\title{
Clustering using K-Means and Fuzzy C-Means on Food Productivity
}

\author{
Adriyendi \\ IAIN Batusangkar, Indonesia \\ elektronikpos@gmail.com
}

\begin{abstract}
This paper provided an overview of analysis and implementation clustering for food productivity. Food productivity is determined by food production. Rice is one of staple food in Indonesia. Rice production is influencing adequacy level of national food production. Rice productivity is very important to accomplishment food affordability. Rice productivity per province in Indonesia must be increased, because large population and high consumption. Rice productivity that fluctuates and tends to decrease, need to clustering to determinant category cluster of productivity. Clustering is using K-Means and Fuzzy C-Means. Method improvement of K-Means is modification Intra Cluster Distance and Inter Cluster Distance. Calculate distance (Inter Cluster Distance and Intra Cluster Distance) to evaluate the clustering results and to compare the efficiency of the clustering algorithms. Method improvement of Fuzzy C-Means is modification algorithm, alternative process and iteration. Data processing is using Excel software. Clustering produce three cluster $\left(C_{1}, C_{2}, C_{3}\right)$ is convergence. Measurement cluster based on comparison of membership cluster, consistency, and productivity. Membership cluster, there is point data anomaly $\left(x_{22}, x_{23}, x_{29}, x_{33}\right)$. Consistency data on K-Means $\left(C_{1}=\right.$ $\left.72.73 \%, C_{2}=93.75 \%, C_{3}=100 \%\right)$. Consistency data on Fuzzy C-Means $\left(C_{1}=100 \%, C_{2}\right.$ $=88.33 \%, C_{3}=87.50 \%$ ). Rice Productivity is Cluster 1 (decrease), Cluster 2 (decrease, except 3 provinces), and Cluster 3 (increase, except 1 province). Majority in rice productivity is $70.59 \%$. Result of clustering showed that majority rice productivity on category cluster is low productivity.
\end{abstract}

Keywords: Clustering, K-Means, Fuzzy C-Means, Food, Rice Productivity

\section{Introduction}

Food production per province in Indonesia influence national food production. Rice is one of staple food in Indonesia. Rice production is determining food productivity. Based on BPS Publication 2015, growth 2015 over 2014 about rice productivity is fluctuates and tends to decrease. Fluctuation in rice productivity is not good for national food production. Good productivity influence adequacy of national food production. Rice productivity on high level is a determinant factor of food affordability. Hence, need to clustering dataset to determinant low productivity and high productivity on rice production per province in Indonesia. Clustering is the process of grouping data objects into similar classes for finding similarities in data and putting similar data into groups namely cluster. Cluster is a collection of data object that are similar in same class and dissimilar to object in other class [1]. Clustering analysis is one of the important technologies in data mining, machine learning, pattern recognition, and many applications. Fuzzy clustering will be a better choice for the data points and clustering algorithms allocated each object to a cluster became most fundamental [2]. K-Means is clustering algorithm that fast, robust, relatively efficient in computational time, simple to implement, and gives comparatively good results if clusters in datasets are distinct or well separated in clustering [3]. K-Means is high 
simplicity, fast convergence rate, efficiency, excellent especially dealing with large datasets, local search ability, better performance for spherical cluster, data samples from variant cluster show obvious different, and practical clustering algorithm in many applications [4]. Fuzzy C-Means is a clustering method similar to K-Means by using fuzzy theory to improve clustering results. Fuzzy C-Means is most popular fuzzy clustering method, easily implemented, has obtained satisfactory results, and become an important tool in many applications [5]. Fuzzy C-Means is very effective in image segmentation and clustering algorithm. [6].

\section{Related Work}

Based on paper [7], clustering using cluster center based K-Means and representative object based Fuzzy C-Means. Fuzzy C-Means Clustering produces close results to KMeans Clustering, but it still requires more computation time than K-Means Clustering. The time complexity of the K-Means Algorithm is $\mathrm{O}$ (ncdi) and the time complexity of Fuzzy C-Means Algorithm is O (ndc2i). It is meaning that K-Means Algorithm seems to be superior then Fuzzy C-Means Algorithm. Based on paper [8], clustering is using K-Means, Fuzzy C-Means, and Possibilistic Fuzzy C-Means for segment in standard and color image. Result shown that Possibilistic Fuzzy C-Means favorable over Fuzzy C-Means and K-Means and provide the better result on noise gray scale images, but it require more computational time than K-Means and Fuzzy C-Means. Based on paper [9], K-Means Algorithm is enough to extract type of tumors from the brain cells, segmentation using Fuzzy C Means for accurate tumor shape extraction of malignant tumor, and both method is gives more accurate result.

In this paper [10] clustering algorithm is K-Means and Fuzzy C-Means. As the number of records increases the time execution of both the technique gets increased but the Fuzzy C-Means performance is found to be better than K-Means Algorithm. The precision, recall and $\mathrm{f}$ measure values are more accurate on applying Fuzzy CMeans compared to K-Means Algorithm. The number of data points is evenly distributed in Fuzzy C-Means Algorithm. In this paper [11], a comparative research between Fuzzy Clustering Algorithm and Hard Clustering Algorithm. Fuzzy CMeans is chosen on the behalf of Fuzzy Clustering Algorithm and K-Means Algorithm is chosen on the behalf of Hard Clustering Algorithm. On the basis of experiments, found that the computational time of K-Means Algorithm is less than that of Fuzzy C-Means Algorithm for the Iris Dataset. This research concludes that the K-Means performance is better than Fuzzy C-Means performance in terms computational time.

\section{Method Proposed}

\subsection{Framework}

Method proposed can be depictured in framework shown on Figure 1.

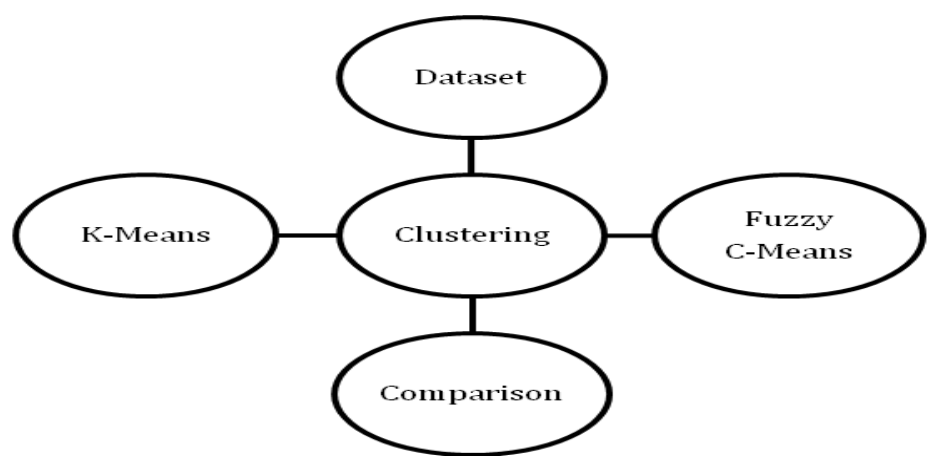




\section{Figure 1. Framework of Clustering}

Figure 1, shown framework of clustering using K-Means and Fuzzy C-Means. Dataset based on data source from BPS Publication 2015. Comparison is between K-Means and Fuzzy C-Means based on Membership Cluster, Consistency Data, and Productivity.

Algorithmic steps for K-Means Clustering [11]:

1. Select $K$ points as initial cluster center (centroid).

2. Repeat:

3. Form $\mathrm{k}$ clusters by assigning all points to the closest cluster center (centroid).

4. Recompute the cluster center (centroid) of each cluster.

5. Until the cluster center (centroid) do not change.

Advantages of K-Means Clustering:

1. K-Means Clustering is a method of vector quantization that is popular for cluster analysis and works great if clusters are spherical.

2. K-Means Clustering tends to find clusters of comparable spatial extent, while the expectation maximization mechanism allows clusters to have different shapes.

3. K-Means Clustering aims to partition $\mathrm{n}$ observations into $\mathrm{k}$ clusters in which each observation belongs to the cluster with the nearest mean, serving as a prototype of the cluster. This algorithm aims at minimizing an objective function.

4. K-Means Clustering is one of the simplest algorithm which uses un-supervised learning method to solve known clustering issues.

5. K-Means Clustering works really well with large datasets.

6. K-Means Clustering may produce tighter clusters than hierarchical clustering, especially if the clusters are globular.

7. K-Means Clustering is most of the times computationally faster than hierarchical clustering for a large number of variables, if keep $\mathrm{k}$ smalls.

8. K-Means Clustering is faster, because order of time complexity is linear with the number of data.

Algorithmic steps of Fuzzy C-Means Clustering [11]:

1. Initialize $U, \mu_{i k} k, m, t, P_{t}$, maximum iteration (treshold) .

2. Select $\mathrm{m}$, initialize the membership function value.

3. Compute the cluster centers.

4. Compute Euclidean distance.

5. Update the membership function.

6. If not converged, go to step 2 .

Advantages of Fuzzy C-Means Clustering:

1. Fuzzy C-Means Clustering is un-supervised learning.

2. Gives best result for overlapped dataset and comparatively better then K-Means Algorithm in process of clustering.

3. Unlike K-Means Clustering, where data point must exclusively, belong to one cluster center, here data point is assigned membership to each cluster center, as a result of which data point may belong to more than one cluster center.

4. Fuzzy C-Means Clustering works with all data is convergences.

\subsection{K-Means}

K-Means Algorithm can be depictured as flowchart for this application, shown on Figure 2. 


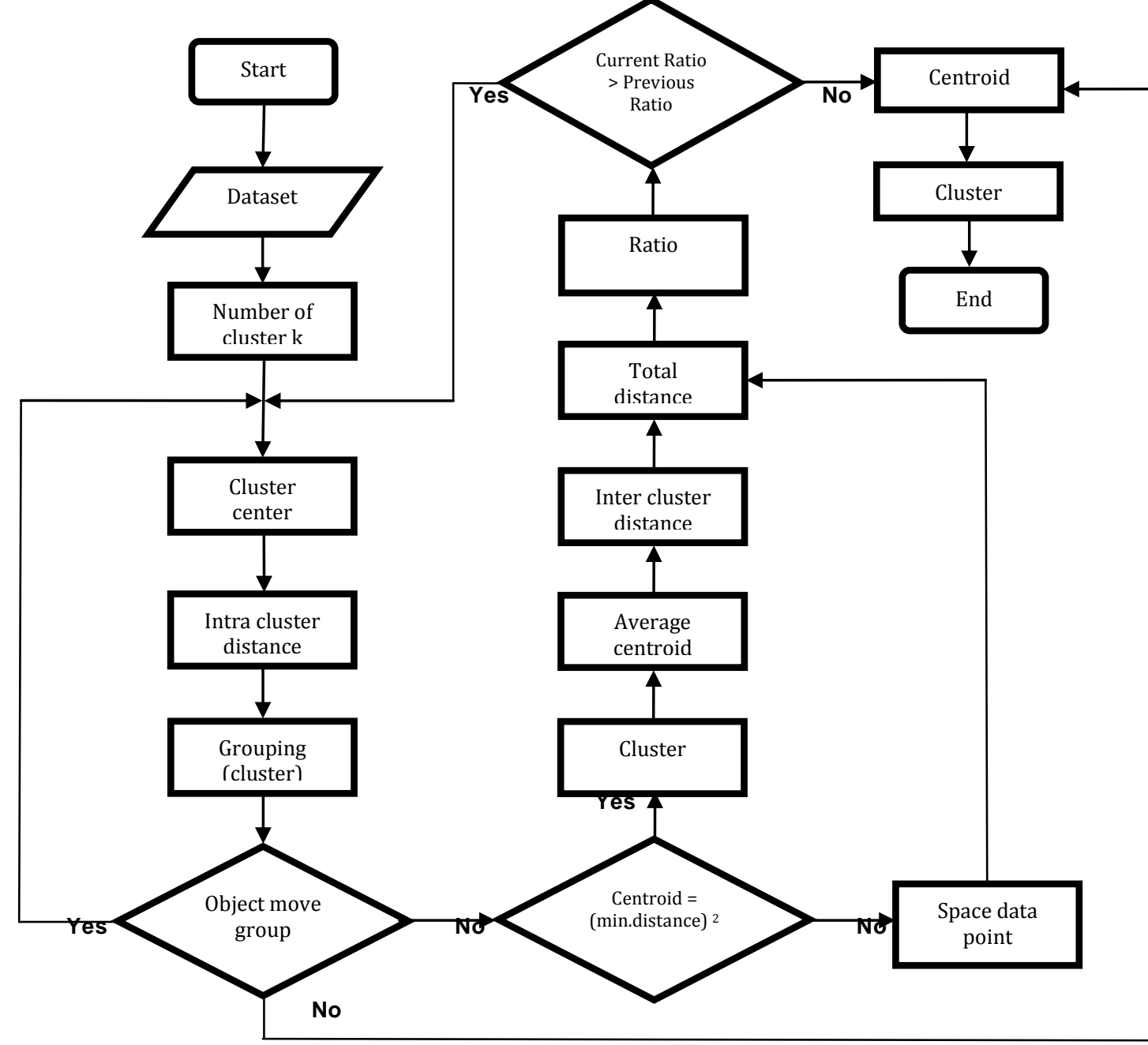

Figure 2. Flowchart K-Means Algorithm

\subsection{Method Improvement of K-Means}

- Intra cluster distance [12] specifies between the data vector within a cluster using Equation (1).

$$
D_{e}=\sqrt{\left(x_{i}-s_{i}\right)^{2}+\left(y_{i}-t_{i}\right)^{2}} ; D=\frac{1}{c} \mid \sum_{i=1}^{c} \sum_{k=1}^{n}\left\|x_{k}-v_{i}\right\|^{2}
$$

with $D_{e}$ is Euclidean distance, $i$ is number of object, $(x, y)$ is coordinate of object, $(s, t)$ is coordinate of cluster center.

- Inter cluster distance [13] is calculate minimum distance between inter cluster center for measure separation cluster using Equation (2).

$$
D_{i j}=\left\|z_{i}-z_{j}\right\| ; D_{\text {inter }}=\left\|v_{i}-v_{j}\right\|
$$

with $D_{i j}$ is distance inter cluster center, $z_{i}$ is cluster center $i$, dan $z_{j}$ is cluster center $j, v_{i}$ is centroids $i$ and $v_{j}$ is centroids $j$. Measurement weighted exponent $(1 \leq m<\infty$, give good result: $1.5 \leq m \leq 3.0)$, cluster $(2 \leq c<n)$, observation data $(1 \leq k \leq N)$, and number of $\operatorname{object}(1 \leq i \leq c)$.

\subsection{Fuzzy C-Means}


Fuzzy C-Means Algorithm can be depictured as flowchart for this application, display on Figure 3.

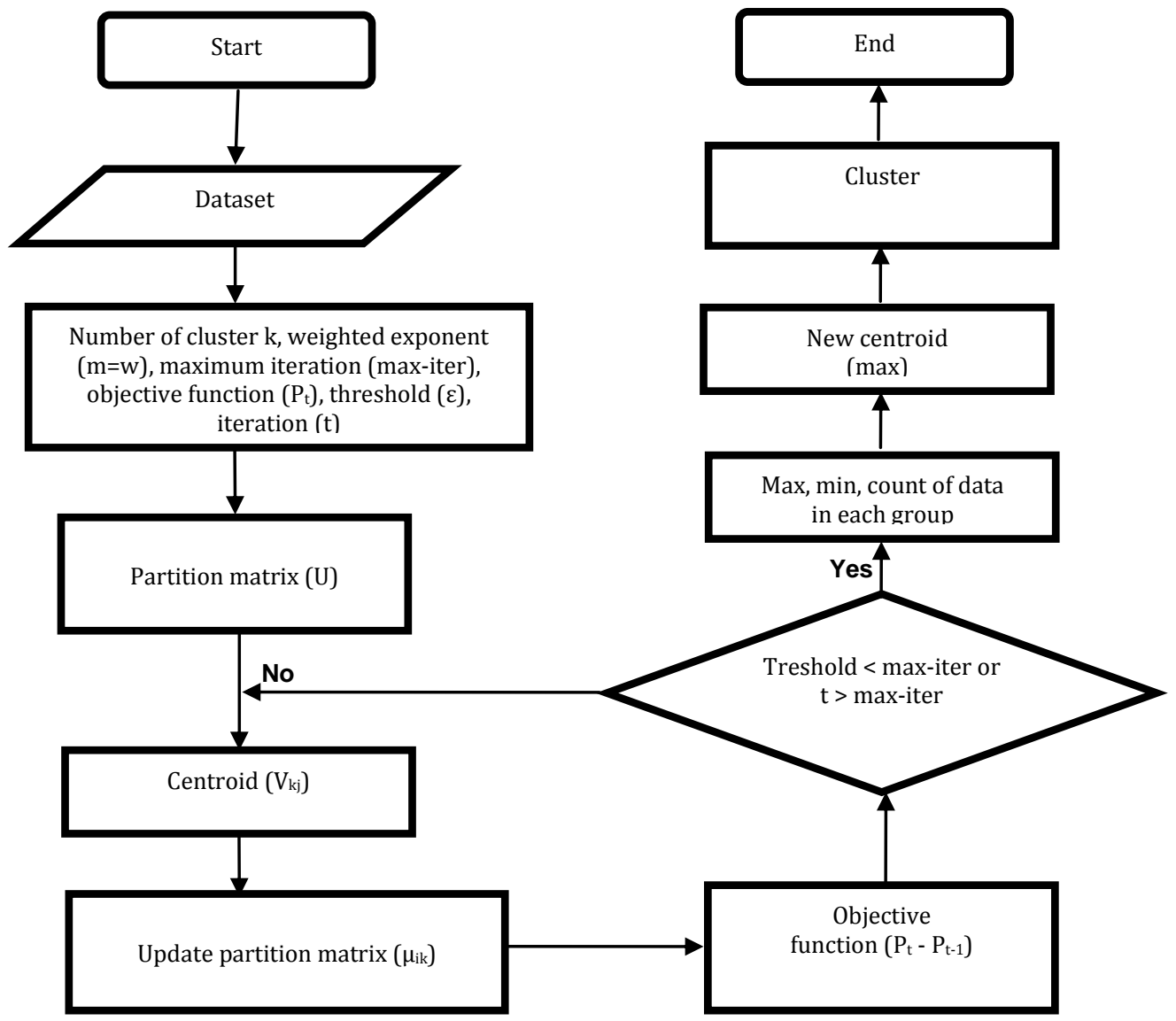

Figure 3. Flowchart Fuzzy C-Means Algorithm

Step by step of Fuzzy C-Means Algorithm based on paper [14]:

- Data $(X) \rightarrow$ matrix (n x m), $X_{i j} \rightarrow($ data $i=1,2,3, \ldots, n$; attribute $j=1,2,3, \ldots, m)$.

- Constrain $\rightarrow$ number of cluster $\mathrm{k}$, weighted exponent (fuzzier $=\mathrm{w}$ or $\mathrm{m}$ ), maximum iteration $\left(\max\right.$-iter), minimum error (threshold $\left.=10^{-6}\right)$, objective function $\left(\mathrm{P}_{\mathrm{t}}\right)$ and iteration $(\mathrm{t}=1)$.

- Initialize $U=\left[\mu_{i j}\right]$, partition matrix $U^{(0)}$ and generate random number $\left(\mu_{\mathrm{ci}}\right) \rightarrow \mathrm{i}=1,2$, $\ldots, \mathrm{n} ; \mathrm{k}=1,2, \ldots, \mathrm{c}$; using Equation (3).

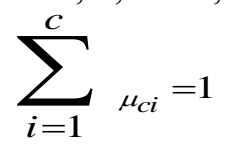

- Calculation matrix of cluster center (centroid); $V_{k j}(\mathrm{k}=1,2 \ldots, \mathrm{c} ; \mathrm{j}=1,2 \ldots, \mathrm{m})$ $V_{k j}=\frac{\sum_{i=1}^{n}\left(\left(_{\mu_{i k}}\right)^{w_{*} X_{i j}}\right)}{\sum_{i=1}^{n}\left(\left(_{\mu_{i k}}\right)^{w}\right)}$

- Calculation objective function $\left(P_{t}\right)$ using Equation (5).

$\boldsymbol{P}_{t}=\sum_{i=1}^{n} \sum_{k=1}^{c}\left(\left[\sum_{j=1}^{m}\left(X_{i j}-V_{k j}\right)^{2}\right]\left({ }_{\mu_{i k}}\right)^{w}\right)$ 
- Update partition matrix $\left(\mu_{i k}\right)$ using Equation (6).

$$
\mu_{i k}=\frac{\left[\sum_{i=1}^{m}\left(x_{i j}-V_{k j}\right)^{2}\right]^{\frac{-1}{w-1}}}{\sum_{k=1}^{c}\left[\sum_{j=1}^{m}\left(x_{i j}-V_{k j}\right)^{2}\right]^{-1}}
$$

- Stopping iteration:

- $\Delta=U^{t}-U^{t-1}$; if $\Delta<$ threshold $(\varepsilon)$, then stop iteration;

- If $\left(\left|P_{t}-P_{t-1}\right|\right)<\varepsilon$ or ( $\mathrm{t}>$ max-iter $)$, then stop iteration;

- If no, then $\mathrm{t}=\mathrm{t}+1$; recalculated centroid $\left(V_{k j}\right)$.

\subsection{Method Improvement of Fuzzy C-Means}

Fuzzy C-Means Clustering modified in this application shown in Table 1.

\section{Table 1. Fuzzy C-Means Clustering}

\begin{tabular}{|c|c|c|}
\hline Algorithm & Application & Stop Iteration \\
\hline $\begin{array}{l}-t \leftarrow 0 \\
- \text { repeat } \\
\quad-t \leftarrow t+1 \\
-U_{t}=F_{\partial}\left(V_{t-1}\right) \\
-V_{t}=G_{\partial}\left(U_{t-1}\right) \\
- \text { until } \\
-(t=T \text { or } \\
\left.\| V_{t^{-}} V_{t-1}\right) \| \leq \varepsilon \\
-(U, V) \leftarrow\left(U_{t}, V_{t}\right)\end{array}$ & $\begin{array}{l}-\mu \rightarrow \mu^{2} \rightarrow \Sigma\left(\mu^{2}\right) \\
-\mu^{2} \cdot x_{i} \rightarrow \Sigma\left(\mu^{2} . x_{i}\right) \\
-\Sigma\left(\mu^{2} \cdot x_{i}\right) / \Sigma\left(\mu^{2}\right) \rightarrow V_{k j} \\
-V_{k j} \rightarrow X_{i j} \cdot V_{k j} \\
-X_{i j} V_{k j} \cdot \mu^{2} \rightarrow L \rightarrow \Sigma(L) \\
-\left(X_{i j \cdot} \cdot V_{k j}\right)^{-1 / w-1} \rightarrow T \rightarrow \Sigma(T)-\Sigma(L . T) \\
-P_{t} \rightarrow \Sigma\left(X_{i j} \cdot V_{k j}\right) \cdot \Sigma(L) \Sigma(L . T) \\
-\left(\mu_{i k}\right) \rightarrow \Sigma\left(X_{i j} \cdot V_{k j}\right)^{-1 / w-1} / \\
\Sigma\left(X_{i j} \cdot V_{k j}\right)^{-1 / w-1}\end{array}$ & $\begin{array}{l}\Delta=U^{t}-U^{t-1} ; \\
\text { - if } \Delta<\text { threshold }(\varepsilon), \\
\quad \text { then stop iteration; } \\
\text { - If }\left(\left|P_{t}-P_{t-1}\right|\right)<\varepsilon \text { or } \\
\quad(t>\text { max-iter }), \\
\quad \text { then stop iteration; } \\
\text { - If not, then } t=t+1 ; \\
\text { recalculated }\left(V_{k j}\right) \\
\text { update }\left(\mu_{i k}\right)\end{array}$ \\
\hline
\end{tabular}

\section{Result and Discussion}

\subsection{Dataset}

The process of clustering using Dataset based on Data Source (Rice Productivity per Province in Publication BPS 2015), display on Table 2.

Table 2. Data Source

\begin{tabular}{|c|c|c|c|c|c|c|c|c|c|}
\hline \multirow[b]{2}{*}{$\left(\mathrm{x}_{\mathrm{i}}\right)$} & \multirow[b]{2}{*}{ Province } & \multicolumn{2}{|c|}{ Year $(\mathrm{Ku} / \mathrm{Ha})$} & \multirow{2}{*}{$\begin{array}{c}\text { Growth } \\
2015 \\
\text { over } 2014 \\
(\%) \\
\end{array}$} & \multirow[b]{2}{*}{$\left(\mathrm{x}_{\mathrm{i}}\right)$} & \multirow[b]{2}{*}{ Province } & \multicolumn{2}{|c|}{ Year $(\mathrm{Ku} / \mathrm{Ha})$} & \multirow{2}{*}{$\begin{array}{c}\text { Growth } \\
2015 \\
\text { over } 2014 \\
(\%)\end{array}$} \\
\hline & & $\begin{array}{c}2014 \\
(\%)\end{array}$ & $\begin{array}{c}2015 \\
(\%)\end{array}$ & & & & $\begin{array}{c}2014 \\
(\%)\end{array}$ & $\begin{array}{c}2015 \\
(\%)\end{array}$ & \\
\hline $\mathrm{x}_{1}$ & Aceh & 48,39 & 50,56 & 4,48 & $\mathrm{X}_{18}$ & NTB & 48,80 & 51,71 & 5,96 \\
\hline $\mathrm{X}_{2}$ & Sumatera Utara & 50,62 & 51,74 & 2,21 & $\mathrm{X}_{19}$ & NTT & 33,46 & 35,61 & 6,43 \\
\hline $\mathrm{X}_{3}$ & Sumatera Barat & 50,06 & 50,25 & 0,38 & $\mathrm{X}_{20}$ & Kalimantan Barat & 30,35 & 29,40 & $-3,13$ \\
\hline $\mathrm{X}_{4}$ & Riau & 36,35 & 36,63 & 0,77 & $\mathrm{X}_{21}$ & KalimantanTengah & 34,57 & 35,07 & 1,45 \\
\hline $\mathrm{X}_{5}$ & Jambi & 45,53 & 44,31 & $-2,68$ & $\mathrm{X}_{22}$ & KalimantanSelatan & 42,05 & 41,87 & $-0,43$ \\
\hline $\mathrm{x}_{6}$ & SumateraSelatan & 45,26 & 48,67 & 7,53 & $\mathrm{X}_{23}$ & Kalimantan Timur & 42,55 & 41,20 & $-3,17$ \\
\hline $\mathrm{X}_{7}$ & Bengkulu & 40,20 & 44,92 & 11,74 & $\mathrm{X}_{24}$ & Kalimantan Utara & 36,05 & 27,27 & $-24,36$ \\
\hline $\mathrm{X}_{8}$ & Lampung & 51,18 & 51,49 & 0,61 & $\mathrm{X}_{25}$ & Sulawesi Utara & 48,91 & 49,05 & 0,29 \\
\hline $\mathrm{X}_{9}$ & BangkaBelitung & 23,62 & 22,85 & $-3,26$ & $\mathrm{X}_{26}$ & Sulawesi Tengah & 46,54 & 48,57 & 4,36 \\
\hline $\mathrm{x}_{10}$ & Kepulauan Riau & 36,44 & 36,46 & 0,05 & $\mathrm{X}_{27}$ & Sulawesi Selatan & 52,17 & 52,41 & 0,46 \\
\hline $\mathrm{X}_{11}$ & DKI Jakarta & 53,86 & 55,95 & 3,88 & $\mathrm{X}_{28}$ & Sulawesi Tenggara & 46,84 & 47,07 & 0,49 \\
\hline $\mathrm{X}_{12}$ & Jawa Barat & 58,82 & 61,22 & 4,08 & $\mathrm{X}_{29}$ & Gorontalo & 50,20 & 55,51 & 10,58 \\
\hline $\mathrm{X}_{13}$ & Jawa Tengah & 53,57 & 60,25 & 12,47 & $\mathrm{X}_{30}$ & Sulawesi Barat & 47,65 & 49,41 & 3,69 \\
\hline $\mathrm{X}_{14}$ & DI Yogyakarta & 57,87 & 60,65 & 4,80 & $\mathrm{X}_{31}$ & Maluku & 47,52 & 55,72 & 17,26 \\
\hline $\mathrm{X}_{15}$ & Jawa Timur & 59,81 & 61,13 & 2,21 & $\mathrm{X}_{32}$ & Maluku Utara & 34,01 & 35,11 & 3,23 \\
\hline $\mathrm{X}_{16}$ & Banten & 52,95 & 56,61 & 6,91 & $\mathrm{X}_{33}$ & Papua Barat & 40,21 & 42,12 & 4,75 \\
\hline $\mathrm{X}_{17}$ & Bali & 60,12 & 62,14 & 3,36 & $\mathrm{X}_{34}$ & Papua & 43,09 & 43,95 & 2,00 \\
\hline & Indonesia & 51,35 & 53,41 & 4,01 & & Indonesia & 51,35 & 53,41 & 4,01 \\
\hline
\end{tabular}


Table 2, shown Dataset $\left(\mathrm{x}_{\mathrm{i}}\right)$ about National Food (Rice Productivity) per province $\left(\mathrm{x}_{\mathrm{i}}=34\right.$ province), measure $(\mathrm{Ku} / \mathrm{Ha}$ : Kuintal $/$ Hectare $=100 \mathrm{~kg} / \mathrm{Ha})$, year $(2014$ and 2015 in \%), growth (2015 over 2014 in \%), and Indonesia (average of national food in \%).

\subsection{K-Means}

Step 1: Dataset based on Data Source in Table 2, can be seen in Table 4.

Step 2: Number of cluster $\mathrm{k}\left(\mathrm{C}_{\mathrm{j}}=3\right)$ display on Table 3 .

Table 3. Cluster for K-Means

\begin{tabular}{|l|l|l|l|l|}
\hline \multicolumn{3}{|l|}{ Cluster } & Description \\
\hline $\mathrm{C}_{\mathrm{i}}$ & $\mathrm{x}_{\mathrm{i}}$ & $\mathrm{z}_{\mathrm{i}}$ & $\mathrm{z}_{\mathrm{j}}$ & Cluster $\left(\mathrm{C}_{\mathrm{i}}\right)=3$ \\
\hline $\mathrm{C}_{1}$ & $\mathrm{x}_{3}$ & 50.06 & 50.25 & Data $\left(\mathrm{x}_{\mathrm{i}}\right)=\mathrm{x}_{3}, \mathrm{x}_{8}, \mathrm{x}_{27}$ (random) \\
\hline $\mathrm{C}_{2}$ & $\mathrm{x}_{8}$ & 51.18 & 51.49 & Centroid Cluster $\left(\mathrm{z}_{\mathrm{i}}\right)$ \\
\hline $\mathrm{C}_{3}$ & $\mathrm{x}_{27}$ & 52.17 & 52.41 & Centroid Cluster $\left(\mathrm{z}_{\mathrm{j}}\right)$ \\
\hline
\end{tabular}

Step 3: Cluster center or centroid (randomly: $\mathrm{x}_{3}, \mathrm{x}_{8}, \mathrm{x}_{27}$ ) can be seen in Table 3. Next centroid based on minimum distance in each cluster.

Step 4: Intra cluster distance using Equation (1) showed on Table 4. Implementing in Excel $=\operatorname{SQRT}\left((\$ C \$ 6-\$ C 44)^{\wedge} 2+(\$ D \$ 6-\$ D 44)^{\wedge} 2\right)$.

Step 5: Grouping based on centroid display on Table 4. Grouping using Excel $=\mathrm{IF}\left(\mathrm{F} 51=" \mathrm{C} 1 ", \$ \mathrm{C} \$ 6, "{ }^{\prime}\right)$. Grouping based on minimum distance using Excel $=\mathrm{IF}(\mathrm{MIN}(\mathrm{C} 51: \mathrm{E} 51)=\mathrm{C} 51, \mathrm{C} \$ 49, \mathrm{IF}(\mathrm{MIN}(\mathrm{C} 51: \mathrm{E} 51)=\mathrm{D} 51, \mathrm{D} \$ 49, \mathrm{IF}(\mathrm{MIN}(\mathrm{C} 51: \mathrm{E} 51)=\mathrm{E} 51, \mathrm{E} \$$ $49, " ')))$. If data point move group, back to step 3. If no move group, next step 6.

Step 6: If centroid cluster $=(\text { minimum distance })^{2}$, then group in new cluster. Minimum distance using Excel $=\mathrm{MIN}(\mathrm{C} 51: \mathrm{E} 51)$. Minimum distance ${ }^{2}$ using Excel $=\mathrm{G} 51 * \mathrm{G} 51$. Total minimum distance (wcv) using Excel $=\mathrm{SUM}(\mathrm{H} 51: \mathrm{H} 84$ ), display on Table 4.

Step 7: Average centroid using Excel =AVERAGEIF(I51:I84,"<>"',I51:I84) in Table 4.

Step 8: Inter cluster distance based on Equation (2) using Excel $=\mathrm{SQRT}((\mathrm{C} 98$ C99)^2+(D98-D99)^2), can be seen in Table 5 .

Step 9: Total distance inter cluster (bcv) using Excel =SUM(C141:C143) in Table 5.

Step 10: Ratio to threshold (bcv/wcv) using Excel $=\mathrm{C} 90 / \mathrm{H} 85$, can be seen in Table 5 .

Step 11: If ratio > threshold or current ratio > previous ratio, then recalculated new centroid using Excel $=\mathrm{IF}(\mathrm{C} 92>\mathrm{C} 93$,"next iteration because current ratio $>$ previous ratio (ratio 1 > ratio 0 )"," current ratio < previous ratio, stop iteration"), shown on Table 5.

Step 12: Iteration back to Step3, to recalculating new centroid until current ratio $\leq$ previous ratio. On iteration 6 , current ratio $\leq$ previous ratio, then stop iteration, shown on Table 6.

Step 13: Cluster determine by grouping data point to centroid based on minimum distance, when current ratio $\leq$ previous ratio. Implementing cluster in Excel $=\mathrm{IF}(\mathrm{MIN}(\mathrm{C} 321: \mathrm{E} 321)=\mathrm{C} 321, \mathrm{C} \$ 49, \operatorname{IF}(\mathrm{MIN}(\mathrm{C} 321: \mathrm{E} 321)=\mathrm{D} 321, \mathrm{D} \$ 49, \operatorname{IF}(\mathrm{MIN}(\mathrm{C} 321: \mathrm{E} 32$ 1) $=\mathrm{E} 321, \mathrm{E} \$ 49, " ')))$, display on Table 7.

Inter-Cluster Distance basically specifies the distance between the cluster center (centroids) of the clusters. The maximum value of Inter-Cluster distance shows that the objects of different clusters are more dissimilar. Intra-Cluster Distance specifies the distance between the data vectors within a cluster. 
Table 4. Distance for K-Means

\begin{tabular}{|c|c|c|c|c|c|c|c|c|c|c|c|c|}
\hline \multirow{2}{*}{$\begin{array}{c}\text { Dataset } \\
\left(\mathrm{x}_{\mathrm{i}}\right)\end{array}$} & \multicolumn{6}{|c|}{ Distance Intra Cluster $\left(\mathrm{D} \mathrm{C} \mathrm{C}_{\mathrm{i}}\right)$} & \multicolumn{2}{|c|}{$\begin{array}{c}\text { Centroid } \\
\left(\mathrm{C}_{1}\right)\end{array}$} & \multicolumn{2}{|c|}{$\begin{array}{c}\text { Centroid } \\
\left(\mathrm{C}_{2}\right)\end{array}$} & \multicolumn{2}{|c|}{$\begin{array}{c}\text { Centroid } \\
\left(\mathrm{C}_{3}\right)\end{array}$} \\
\hline & $\mathrm{D} \mathrm{C}_{1}$ & $\mathrm{D} \mathrm{C}_{2}$ & $\mathrm{D} \mathrm{C}_{3}$ & $\mathrm{C}_{\mathrm{i}}$ & $\min \mathrm{D}$ & $(\min D)^{2}$ & $\mathrm{z}_{\mathrm{i}}$ & $\mathrm{z}_{\mathrm{j}}$ & $\mathrm{z}_{\mathrm{i}}$ & $\mathrm{z}_{\mathrm{j}}$ & $\mathrm{z}_{\mathrm{i}}$ & $\mathrm{z}_{\mathrm{j}}$ \\
\hline $\mathrm{x}_{1}$ & 1.6985 & 2.9409 & 4.2084 & $\mathrm{C}_{1}$ & 1.6985 & 2.8850 & 48.39 & 50.56 & & & & \\
\hline $\mathrm{x}_{2}$ & 1.5918 & 0.6133 & 1.6886 & $\mathrm{C}_{2}$ & 0.6133 & 0.3761 & & & 50.62 & 51.74 & & \\
\hline $\mathrm{x}_{3}$ & 0.0000 & 1.6709 & 3.0196 & $\mathrm{C}_{1}$ & 0.0000 & 0.0000 & 50.06 & 50.25 & & & & \\
\hline $\mathrm{x}_{4}$ & 19.3253 & 20.9940 & 22.3446 & $\mathrm{C}_{1}$ & 19.3253 & 373.4685 & 36.35 & 36.63 & & & & \\
\hline $\mathrm{x}_{5}$ & 7.4702 & 9.1365 & 10.4738 & $\mathrm{C}_{1}$ & 7.4702 & 55.8045 & 45.53 & 44.31 & & & & \\
\hline $\mathrm{x}_{6}$ & 5.0534 & 6.5573 & 7.8572 & $\mathrm{C}_{1}$ & 5.0534 & 25.5364 & 45.26 & 48.67 & & & & \\
\hline $\mathrm{x}_{7}$ & 11.2084 & 12.7955 & 14.1202 & $\mathrm{C}_{1}$ & 11.2084 & 125.6285 & 40.20 & 44.92 & & & & \\
\hline $\mathrm{x}_{8}$ & 1.6709 & 0.0000 & 1.3515 & $\mathrm{C}_{2}$ & 0.0000 & 0.0000 & & & 51.18 & 51.49 & & \\
\hline $\mathrm{X}_{9}$ & 38.0767 & 39.7467 & 41.0962 & $\mathrm{C}_{1}$ & 38.0767 & 1449.8336 & 23.62 & 22.85 & & & & \\
\hline $\mathrm{x}_{10}$ & 19.3822 & 21.0516 & 22.4017 & $\mathrm{C}_{1}$ & 19.3822 & 375.6685 & 36.44 & 36.46 & & & & \\
\hline $\mathrm{x}_{11}$ & 6.8505 & 5.2033 & 3.9227 & $\mathrm{C}_{3}$ & 3.9227 & 15.3877 & & & & & 53.86 & 55.95 \\
\hline $\mathrm{x}_{12}$ & 14.0385 & 12.3710 & 11.0381 & $\mathrm{C}_{3}$ & 11.0381 & 121.8386 & & & & & 58.82 & 61.22 \\
\hline $\mathrm{x}_{13}$ & 10.5981 & 9.0802 & 7.9640 & $\mathrm{C}_{3}$ & 7.9640 & 63.4256 & & & & & 53.57 & 60.25 \\
\hline $\mathrm{x}_{14}$ & 13.0060 & 11.3429 & 10.0194 & $\mathrm{C}_{3}$ & 10.0194 & 100.3876 & & & & & 57.87 & 60.65 \\
\hline $\mathrm{x}_{15}$ & 14.6095 & 12.9386 & 11.5934 & $\mathrm{C}_{3}$ & 11.5934 & 134.4080 & & & & & 59.81 & 61.13 \\
\hline $\mathrm{x}_{16}$ & 6.9858 & 5.4173 & 4.2718 & $\mathrm{C}_{3}$ & 4.2718 & 18.2484 & & & & & 52.95 & 56.61 \\
\hline $\mathrm{x}_{17}$ & 15.5748 & 13.9049 & 12.5648 & $\mathrm{C}_{3}$ & 12.5648 & 157.8754 & & & & & 60.12 & 62.14 \\
\hline $\mathrm{x}_{18}$ & 1.9285 & 2.3901 & 3.4419 & $\mathrm{C}_{1}$ & 1.9285 & 3.7192 & 48.80 & 51.71 & & & & \\
\hline $\mathrm{x}_{19}$ & 22.1334 & 23.7944 & 25.1457 & $\mathrm{C}_{1}$ & 22.1334 & 489.8896 & 33.46 & 35.61 & & & & \\
\hline $\mathrm{x}_{20}$ & 28.6916 & 30.3621 & 31.7108 & $\mathrm{C}_{1}$ & 28.6916 & 823.2066 & 30.35 & 29.40 & & & & \\
\hline $\mathrm{x}_{21}$ & 21.6881 & 23.3561 & 24.7070 & $\mathrm{C}_{1}$ & 21.6881 & 470.3725 & 34.57 & 35.07 & & & & \\
\hline $\mathrm{x}_{22}$ & 11.5924 & 13.2628 & 14.6118 & $\mathrm{C}_{1}$ & 11.5924 & 134.3845 & 42.05 & 41.87 & & & & \\
\hline $\mathrm{x}_{23}$ & 11.7602 & 13.4299 & 14.7719 & $\mathrm{C}_{1}$ & 11.7602 & 138.3026 & 42.55 & 41.20 & & & & \\
\hline $\mathrm{x}_{24}$ & 26.9139 & 28.5574 & 29.8643 & $\mathrm{C}_{1}$ & 26.9139 & 724.3605 & 36.05 & 27.27 & & & & \\
\hline $\mathrm{x}_{25}$ & 1.6621 & 3.3326 & 4.6816 & $\mathrm{C}_{1}$ & 1.6621 & 2.7625 & 48.91 & 49.05 & & & & \\
\hline $\mathrm{x}_{26}$ & 3.9004 & 5.4823 & 6.8149 & $\mathrm{C}_{1}$ & 3.9004 & 15.2128 & 46.54 & 48.57 & & & & \\
\hline $\mathrm{x}_{27}$ & 3.0196 & 1.3515 & 0.0000 & $\mathrm{C}_{3}$ & 0.0000 & 0.0000 & & & & & 52.17 & 52.41 \\
\hline $\mathrm{x}_{28}$ & 4.5256 & 6.1945 & 7.5448 & $\mathrm{C}_{1}$ & 4.5256 & 20.4808 & 46.84 & 47.07 & & & & \\
\hline $\mathrm{x}_{29}$ & 5.2619 & 4.1377 & 3.6730 & $\mathrm{C}_{3}$ & 3.6730 & 13.4909 & & & & & 50.20 & 55.51 \\
\hline $\mathrm{x}_{30}$ & 2.5522 & 4.0972 & 5.4250 & $\mathrm{C}_{1}$ & 2.5522 & 6.5137 & 47.65 & 49.41 & & & & \\
\hline $\mathrm{x}_{31}$ & 6.0310 & 5.5936 & 5.7078 & $\mathrm{C}_{2}$ & 5.5936 & 31.2885 & & & 47.52 & 55.72 & & \\
\hline $\mathrm{x}_{32}$ & 22.0640 & 23.7300 & 25.0814 & $\mathrm{C}_{1}$ & 22.0640 & 486.8221 & 34.01 & 35.11 & & & & \\
\hline $\mathrm{x}_{33}$ & 12.7718 & 14.4270 & 15.7774 & $\mathrm{C}_{1}$ & 12.7718 & 163.1194 & 40.21 & 42.12 & & & & \\
\hline $\mathrm{x}_{34}$ & 9.3953 & 11.0589 & 12.4104 & $\mathrm{C}_{1}$ & 9.3953 & 88.2709 & 43.09 & 43.95 & & & & \\
\hline \multicolumn{6}{|c|}{ Total minimum distance ${ }^{2}$ (wcv) } & 6632.9695 & \multicolumn{6}{|c|}{ average centroid $\left(\mathrm{z}_{\mathrm{i}}, \mathrm{z}_{\mathrm{j}}\right)$} \\
\hline & & & & & & & 40.95 & 41.46 & 49.77 & 52.98 & 55.49 & 58.43 \\
\hline
\end{tabular}

Highly superior clusters have high intra-class similarity (intra cluster distance) and low inter-class similarity (inter cluster distance). 
Table 5. Ratio for K-Means

\begin{tabular}{|l|l|l|l|l|l|l|l|}
\hline \multicolumn{2}{|l|}{ Inter cluster distance (D) } & $\mathrm{C}_{\mathrm{i}}$ & $\mathrm{z}_{\mathrm{i}}$ & $\mathrm{z}_{\mathrm{j}}$ & $\mathrm{C}_{\mathrm{i}}$ & $\mathrm{z}_{\mathrm{i}}$ & $\mathrm{z}_{\mathrm{j}}$ \\
\hline $\mathrm{C}_{1}-\mathrm{C}_{2}$ & 1.6709 & $\mathrm{C}_{1}$ & 40.95 & 41.46 & $\mathrm{C}_{1}$ & 40.95 & 41.46 \\
\hline $\mathrm{C}_{1}-\mathrm{C}_{3}$ & 3.0196 & $\mathrm{C}_{2}$ & 49.77 & 52.98 & $\mathrm{C}_{2}$ & 49.77 & 52.98 \\
\hline $\mathrm{C}_{2}-\mathrm{C}_{3}$ & 1.3515 & $\mathrm{C}_{3}$ & 55.49 & 58.43 & $\mathrm{C}_{3}$ & 55.49 & 58.43 \\
\hline bcv & 6.0420 & ratio 1 & 0.0009 & \multicolumn{3}{|c}{ ratio $1>$ ratio 0,} \\
bcv & 6632.9695 & ratio 0 & 0 & \multicolumn{3}{|c|}{ next iteration } \\
\hline
\end{tabular}

Table 6. Iteration for K-Means

\begin{tabular}{|c|c|c|c|c|c|c|}
\hline \multicolumn{7}{|c|}{ Iteration 1} \\
\hline $\mathrm{C}_{\mathrm{i}}$ & $\mathrm{z}_{\mathrm{i}}$ & $\mathrm{z}_{\mathrm{i}}$ & inter & $\mathrm{D}$ & bcv & WcV \\
\hline $\mathrm{C}_{1}$ & 50.06 & 50.25 & $\mathrm{C}_{1}-\mathrm{C}_{2}$ & 1.6709 & 6.0420 & 6632.9695 \\
\hline $\mathrm{C}_{2}$ & 51.18 & 51.49 & $\mathrm{C}_{1}-\mathrm{C}_{3}$ & 3.0196 & Ratio 1 & 0.0009 \\
\hline $\mathrm{C}_{3}$ & 52.17 & 52.41 & $\mathrm{C}_{2}-\mathrm{C}_{3}$ & 1.3515 & Ratio 0 & 0 \\
\hline \multicolumn{7}{|c|}{ Ratio $1>$ Ratio 0, Next Iterartion } \\
\hline \multicolumn{7}{|c|}{ Iteration 2} \\
\hline $\mathrm{C}_{\mathrm{i}}$ & $\mathrm{Z}_{\mathrm{i}}$ & $\mathrm{z}_{\mathrm{j}}$ & inter & $\mathrm{D}$ & $\mathrm{bcv}$ & WCV \\
\hline $\mathrm{C}_{1}$ & 40.95 & 41.46 & $\mathrm{C}_{1}-\mathrm{C}_{2}$ & 14.5147 & 44.7529 & 1849.0325 \\
\hline $\mathrm{C}_{2}$ & 49.77 & 52.98 & $\mathrm{C}_{1}-\mathrm{C}_{3}$ & 22.3454 & Ratio 2 & 0.0242 \\
\hline $\mathrm{C}_{3}$ & 55.49 & 58.43 & $\mathrm{C}_{2}-\mathrm{C}_{3}$ & 7.8928 & Ratio 1 & 0.0009 \\
\hline \multicolumn{7}{|c|}{ Ratio $2>$ Ratio 1, Next Iterartion } \\
\hline \multicolumn{7}{|c|}{ Iteration 3} \\
\hline $\mathrm{C}_{\mathrm{i}}$ & $\mathrm{Z}_{\mathrm{i}}$ & $z_{j}$ & inter & $\mathrm{D}$ & bcv & WcV \\
\hline $\mathrm{C}_{1}$ & 37.03 & 36.91 & $\mathrm{C}_{1}-\mathrm{C}_{2}$ & 18.2924 & 60.2352 & 1178.1934 \\
\hline $\mathrm{C}_{2}$ & 48.78 & 50.94 & $\mathrm{C}_{1}-\mathrm{C}_{3}$ & 30.1150 & Ratio 3 & 0.0511 \\
\hline $\mathrm{C}_{3}$ & 56.71 & 59.71 & $\mathrm{C}_{2}-\mathrm{C}_{3}$ & 11.8278 & Ratio 2 & 0.0242 \\
\hline \multicolumn{7}{|c|}{ Ratio $3>$ Ratio 2, Next Iterartion } \\
\hline \multicolumn{7}{|c|}{ Iteration 4} \\
\hline $\mathrm{C}_{\mathrm{i}}$ & $\mathrm{Z}_{\mathrm{i}}$ & $\mathrm{z}_{\mathrm{j}}$ & inter & $\mathrm{D}$ & bcv & wcV \\
\hline $\mathrm{C}_{1}$ & 35.82 & 35.71 & $\mathrm{C}_{1}-\mathrm{C}_{2}$ & 18.9171 & 63.6370 & 1111.3304 \\
\hline $\mathrm{C}_{2}$ & 48.18 & 50.03 & $\mathrm{C}_{1}-\mathrm{C}_{3}$ & 31.8183 & Ratio 4 & 0.0573 \\
\hline $\mathrm{C}_{3}$ & 56.71 & 59.71 & $\mathrm{C}_{2}-\mathrm{C}_{3}$ & 12.9016 & Ratio 3 & 0.0511 \\
\hline \multicolumn{7}{|c|}{ Ratio 4 > Ratio 3, Next Iterartion } \\
\hline \multicolumn{7}{|c|}{ Iteration 5} \\
\hline $\mathrm{C}_{\mathrm{i}}$ & $\mathrm{Z}_{\mathrm{i}}$ & $\mathrm{z}_{\mathrm{j}}$ & inter & $\mathrm{D}$ & bcv & WCV \\
\hline $\mathrm{C}_{1}$ & 35.42 & 34.87 & $\mathrm{C}_{1}-\mathrm{C}_{2}$ & 19.2477 & 6.4319 & 1096.2603 \\
\hline $\mathrm{C}_{2}$ & 47.69 & 49.71 & $\mathrm{C}_{1}-\mathrm{C}_{3}$ & 32.7122 & Ratio 5 & 0.0597 \\
\hline $\mathrm{C}_{3}$ & 56.71 & 59.71 & $\mathrm{C}_{2}-\mathrm{C}_{3}$ & 13.4720 & Ratio 4 & 0.0573 \\
\hline \multicolumn{7}{|c|}{ Ratio $5>$ Ratio 4, Next Iterartion } \\
\hline \multicolumn{7}{|c|}{ Iteration 6} \\
\hline $\mathrm{C}_{\mathrm{i}}$ & $\mathrm{Z}_{\mathrm{i}}$ & $\mathrm{z}_{\mathrm{j}}$ & inter & $\mathrm{D}$ & bcv & wcV \\
\hline $\mathrm{C}_{1}$ & 35.42 & 34.87 & $\mathrm{C}_{1}-\mathrm{C}_{2}$ & 19.2477 & 65.4319 & 1096.2603 \\
\hline $\mathrm{C}_{2}$ & 47.69 & 49.71 & $\mathrm{C}_{1}-\mathrm{C}_{3}$ & 32.7122 & Ratio 6 & 0.0597 \\
\hline $\mathrm{C}_{3}$ & 56.71 & 59.71 & $\mathrm{C}_{2}-\mathrm{C}_{3}$ & 13.4720 & Ratio 5 & 0.0597 \\
\hline \multicolumn{7}{|c|}{ Ratio $6 \leq$ Ratio 5, Stop Iterartion } \\
\hline
\end{tabular}

Clustering algorithms have categories: hierarchical-based algorithms, partition-based algorithms, density-based algorithms and grid based algorithms. Partition-based clustering is cluster center (centroid) based which splits data points into $\mathrm{k}$ partition and each partition represents a cluster. K-Means is method can effectively improve the speed and accuracy of clustering, reducing the computational complexity. 
Table 7. Membership Cluster for K-Means

\begin{tabular}{|c|c|c|c|c|c|c|c|c|c|c|}
\hline \multirow{2}{*}{ xi } & \multirow{2}{*}{$\mathrm{C}_{\mathrm{i}}$} & \multicolumn{2}{|c|}{$\mathrm{C}_{1}$} & \multicolumn{2}{|c|}{$\mathrm{C}_{2}$} & \multicolumn{2}{|c|}{$\mathrm{C}_{3}$} & \multirow{2}{*}{$\begin{array}{l}\text { Membership } \\
\text { Cluster }\left(C_{1}\right)\end{array}$} & \multirow{2}{*}{$\begin{array}{l}\text { Membership } \\
\text { Cluster }\left(C_{2}\right)\end{array}$} & \multirow{2}{*}{$\begin{array}{l}\text { Membership } \\
\text { Cluster }\left(C_{3}\right)\end{array}$} \\
\hline & & $\mathrm{z}_{\mathrm{i}}$ & $\mathrm{z}_{\mathrm{j}}$ & $\mathrm{z}_{\mathrm{i}}$ & $\mathrm{z}_{\mathrm{j}}$ & $\mathrm{z}_{\mathrm{i}}$ & $\mathrm{z}_{\mathrm{j}}$ & & & \\
\hline $\mathrm{x}_{1}$ & $\mathrm{C}_{2}$ & & & 48.39 & 50.56 & & & & Aceh & \\
\hline $\mathrm{x}_{2}$ & $\mathrm{C}_{2}$ & & & 50.62 & 51.74 & & & & Sumatera Utara & \\
\hline $\mathrm{x}_{3}$ & $\mathrm{C}_{2}$ & & & 50.06 & 50.25 & & & & Sumatera Barat & \\
\hline $\mathrm{x}_{4}$ & $\mathrm{C}_{1}$ & 36.35 & 36.63 & & & & & Riau & & \\
\hline $\mathrm{x}_{5}$ & $\mathrm{C}_{2}$ & & & 45.53 & 44.31 & & & & Jambi & \\
\hline $\mathrm{x}_{6}$ & $\mathrm{C}_{2}$ & & & 45.26 & 48.67 & & & & Sumatera Selatan & \\
\hline $\mathrm{x}_{7}$ & $\mathrm{C}_{2}$ & & & 40.20 & 44.92 & & & & Bengkulu & \\
\hline $\mathrm{x}_{8}$ & $\mathrm{C}_{2}$ & & & 51.18 & 51.49 & & & & Lampung & \\
\hline $\mathrm{X}_{9}$ & $\mathrm{C}_{1}$ & 23.62 & 22.85 & & & & & Bangka Belitung & & \\
\hline $\mathrm{x}_{10}$ & $\mathrm{C}_{1}$ & 36.44 & 36.46 & & & & & Kepulauan Riau & & \\
\hline $\mathrm{x}_{11}$ & $\mathrm{C}_{3}$ & & & & & 53.86 & 55.95 & & & DKI Jakarta \\
\hline $\mathrm{x}_{12}$ & $\mathrm{C}_{3}$ & & & & & 58.82 & 61.22 & & & Jawa Barat \\
\hline $\mathrm{x}_{13}$ & $\mathrm{C}_{3}$ & & & & & 53.57 & 60.25 & & & Jawa Tengah \\
\hline $\mathrm{x}_{14}$ & $\mathrm{C}_{3}$ & & & & & 57.87 & 60.65 & & & $\begin{array}{l}\text { DI } \\
\text { Yogyakarta }\end{array}$ \\
\hline $\mathrm{x}_{15}$ & $\mathrm{C}_{3}$ & & & & & 59.81 & 61.13 & & & Jawa Timur \\
\hline $\mathrm{x}_{16}$ & $\mathrm{C}_{3}$ & & & & & 52.95 & 56.61 & & & Banten \\
\hline $\mathrm{x}_{17}$ & $\mathrm{C}_{3}$ & & & & & 60.12 & 62.14 & & & Bali \\
\hline $\mathrm{x}_{18}$ & $\mathrm{C}_{2}$ & & & 48.80 & 51.71 & & & & $\begin{array}{l}\text { Nusa Tenggara } \\
\text { Barat }\end{array}$ & \\
\hline $\mathrm{x}_{19}$ & $\mathrm{C}_{1}$ & 33.46 & 35.61 & & & & & $\begin{array}{l}\text { Nusa Tenggara } \\
\text { Timur }\end{array}$ & & \\
\hline $\mathrm{x}_{20}$ & $\mathrm{C}_{1}$ & 30.35 & 29.40 & & & & & Kalimantan Barat & & \\
\hline $\mathrm{x}_{21}$ & $\mathrm{C}_{1}$ & 34.57 & 35.07 & & & & & $\begin{array}{l}\text { Kalimantan } \\
\text { Tengah } \\
\end{array}$ & & \\
\hline $\mathrm{x}_{22}$ & $\mathrm{C}_{1}$ & 42.05 & 41.87 & & & & & $\begin{array}{l}\text { Kalimantan } \\
\text { Selatan }\end{array}$ & & \\
\hline $\mathrm{x}_{23}$ & $\mathrm{C}_{1}$ & 42.55 & 41.20 & & & & & $\begin{array}{l}\text { Kalimantan } \\
\text { Timur }\end{array}$ & & \\
\hline $\mathrm{x}_{24}$ & $\mathrm{C}_{1}$ & 36.05 & 27.27 & & & & & Kalimantan Utara & & \\
\hline $\mathrm{x}_{25}$ & $\mathrm{C}_{2}$ & & & 48.91 & 49.05 & & & & Sulawesi Utara & \\
\hline $\mathrm{x}_{26}$ & $\mathrm{C}_{2}$ & & & 46.54 & 48.57 & & & & Sulawesi Tengah & \\
\hline $\mathrm{x}_{27}$ & $\mathrm{C}_{2}$ & & & 52.17 & 52.41 & & & & Sulawesi Selatan & \\
\hline $\mathrm{x}_{28}$ & $\mathrm{C}_{2}$ & & & 46.84 & 47.07 & & & & $\begin{array}{l}\text { Sulawesi } \\
\text { Tenggara } \\
\end{array}$ & \\
\hline $\mathrm{X}_{29}$ & $\mathrm{C}_{2}$ & & & 50.20 & 55.51 & & & & Gorontalo & \\
\hline $\mathrm{x}_{30}$ & $\mathrm{C}_{2}$ & & & 47.65 & 49.41 & & & & Sulawesi Barat & \\
\hline $\mathrm{x}_{31}$ & $\mathrm{C}_{2}$ & & & 47.52 & 55.72 & & & & Maluku & \\
\hline $\mathrm{x}_{32}$ & $\mathrm{C}_{1}$ & 34.01 & 35.11 & & & & & Maluku Utara & & \\
\hline $\mathrm{x}_{33}$ & $\mathrm{C}_{1}$ & 40.21 & 42.12 & & & & & Papua Barat & & \\
\hline $\mathrm{x}_{34}$ & $\mathrm{C}_{2}$ & & & 43.09 & 43.95 & & & & Papua & \\
\hline
\end{tabular}

Clustering produce 3 clusters. Membership cluster in Cluster 1 is 11 data point. Membership cluster in Cluster 2 is 16 data point. Membership cluster in Cluster 3 is 7 data point. 


\subsection{Fuzzy C-Means}

Initialize Fuzzy C-Means Clustering using Excel software, display on Table 8.

\section{Table 8. Cluster for Fuzzy C-Means}

\begin{tabular}{|c|c|}
\hline Number of cluster $\mathrm{k}$ & 3 \\
\hline Maximum iteration (max-iter) & 100 \\
\hline Weighted exponent $(\mathrm{m}=\mathrm{w})$ & 2 \\
\hline Treshold (minimum error $=\varepsilon)$ & 0.000001 \\
\hline
\end{tabular}

Step 1: Dataset based on Data Source can be seen in Table 2.

Step 2: Number of cluster k (3), max-iter (100), weighted exponent or fuzzier $(m=2)$, threshold $\left(\varepsilon=0.000001\right.$ or $\left.10^{-6}\right)$, objective function $\left(\mathrm{P}_{\mathrm{t}}\right)$ and iteration $(\mathrm{t}=1)$.

Step 3: Random Number (number $>0$ and number $<1$, count $=1$ ) based on Equation (3), using Excel =SUM(K7:M7), can be seen on Table 9.

Table 9. Random Number for Fuzzy C-Means

\begin{tabular}{|c|c|c|c|c|c|c|c|c|c|}
\hline $\mathrm{x}_{\mathrm{i}}$ & $\mathrm{C}_{1}$ & $\mathrm{C}_{2}$ & $\mathrm{C}_{3}$ & Count & $\mathrm{x}_{\mathrm{i}}$ & $\mathrm{C}_{1}$ & $\mathrm{C}_{2}$ & $\mathrm{C}_{3}$ & Count \\
\hline $\mathrm{x}_{1}$ & 0.5 & 0.4 & 0.1 & 1 & $\mathrm{x}_{18}$ & 0.1 & 0.6 & 0.3 & 1 \\
\hline $\mathrm{x}_{2}$ & 0.6 & 0.3 & 0.1 & 1 & $\mathrm{x}_{19}$ & 0.1 & 0.7 & 0.2 & 1 \\
\hline $\mathrm{x}_{3}$ & 0.7 & 0.2 & 0.1 & 1 & $\mathrm{x}_{20}$ & 0.1 & 0.8 & 0.1 & 1 \\
\hline $\mathrm{x}_{4}$ & 0.8 & 0.1 & 0.1 & 1 & $\mathrm{x}_{21}$ & 0.5 & 0.4 & 0.1 & 1 \\
\hline $\mathrm{x}_{5}$ & 0.4 & 0.1 & 0.5 & 1 & $\mathrm{x}_{22}$ & 0.6 & 0.3 & 0.1 & 1 \\
\hline $\mathrm{x}_{6}$ & 0.3 & 0.1 & 0.6 & 1 & $\mathrm{x}_{23}$ & 0.7 & 0.2 & 0.1 & 1 \\
\hline $\mathrm{x}_{7}$ & 0.2 & 0.1 & 0.7 & 1 & $\mathrm{x}_{24}$ & 0.8 & 0.1 & 0.1 & 1 \\
\hline $\mathrm{x}_{8}$ & 0.1 & 0.1 & 0.8 & 1 & $\mathrm{x}_{25}$ & 0.6 & 0.3 & 0.1 & 1 \\
\hline $\mathrm{x}_{9}$ & 0.1 & 0.5 & 0.4 & 1 & $\mathrm{x}_{26}$ & 0.8 & 0.1 & 0.1 & 1 \\
\hline $\mathrm{x}_{10}$ & 0.1 & 0.6 & 0.3 & 1 & $\mathrm{x}_{27}$ & 0.3 & 0.1 & 0.6 & 1 \\
\hline $\mathrm{x}_{11}$ & 0.1 & 0.7 & 0.2 & 1 & $\mathrm{x}_{28}$ & 0.1 & 0.1 & 0.8 & 1 \\
\hline $\mathrm{x}_{12}$ & 0.1 & 0.8 & 0.1 & 1 & $\mathrm{x}_{29}$ & 0.1 & 0.6 & 0.3 & 1 \\
\hline $\mathrm{x}_{13}$ & 0.4 & 0.1 & 0.5 & 1 & $\mathrm{x}_{30}$ & 0.1 & 0.5 & 0.4 & 1 \\
\hline $\mathrm{x}_{14}$ & 0.3 & 0.1 & 0.6 & 1 & $\mathrm{x}_{31}$ & 0.2 & 0.1 & 0.7 & 1 \\
\hline $\mathrm{x}_{15}$ & 0.2 & 0.1 & 0.7 & 1 & $\mathrm{x}_{32}$ & 0.4 & 0.1 & 0.5 & 1 \\
\hline $\mathrm{x}_{16}$ & 0.1 & 0.1 & 0.8 & 1 & $\mathrm{x}_{33}$ & 0.7 & 0.2 & 0.1 & 1 \\
\hline $\mathrm{x}_{17}$ & 0.1 & 0.5 & 0.4 & 1 & $\mathrm{x}_{34}$ & 0.5 & 0.4 & 0.1 & 1 \\
\hline
\end{tabular}

Step 4: Partition matrix $(\mu)$, centroid $\left(\mathrm{V}_{\mathrm{kj}}=\Sigma\left(\mu^{2} . \mathrm{x}_{\mathrm{i}}\right) / \Sigma\left(\mu^{2}\right)\right.$ using Equation (4), display on Table 10, and Table 11.

Table 10. Calculation Centroid for Fuzzy C-Means

\begin{tabular}{|l|l|}
\hline Matrix $(\mu)$ & Excel \\
\hline$\mu^{2}$ & $=$ POWER(B46,\$H\$7) \\
\hline$\Sigma\left(\mu^{2}\right)$ & $=\mathrm{SUM}(\mathrm{F} 46: F 79)$ \\
\hline$\mu^{2} \cdot \mathrm{x}_{\mathrm{i}}$ & $=\mathrm{F} 46 * \mathrm{C} 7$ \\
\hline$\Sigma\left(\mu^{2} \cdot \mathrm{x}_{\mathrm{i}}\right)$ & $=\mathrm{SUM}(\mathrm{J} 46: \mathrm{J} 79)$ \\
\hline$\Sigma\left(\mu^{2} \cdot \mathrm{x}_{\mathrm{i}}\right) / \Sigma\left(\mu^{2}\right)$ & $=\mathrm{J} 81 /$ \$F\$80 \\
\hline
\end{tabular}


Table 11. Centroid for Fuzzy C-Means

\begin{tabular}{|c|c|c|c|c|c|c|c|c|c|c|c|c|}
\hline$x_{i}$ & \multicolumn{3}{|c|}{$\mu$} & \multicolumn{3}{|c|}{$\mu^{2}$} & \multicolumn{2}{|c|}{$\mu^{2} \cdot x_{i}\left(C_{1}\right)$} & \multicolumn{2}{|c|}{$\mu^{2} \cdot x_{i}\left(C_{2}\right)$} & \multicolumn{2}{|c|}{$\mu^{2} \cdot \mathrm{X}_{\mathrm{i}}\left(\mathrm{C}_{3}\right)$} \\
\hline $\mathrm{x}_{1}$ & 0.5 & 0.4 & 0.1 & 0.2500 & 0.1600 & 0.0100 & 12.0975 & 12.6400 & 7.7424 & 8.0896 & 0.4839 & 0.5056 \\
\hline $\mathrm{x}_{2}$ & 0.6 & 0.3 & 0.1 & 0.3600 & 0.0900 & 0.0100 & 18.2232 & 18.6264 & 4.5558 & 4.6566 & 0.5062 & 0.5174 \\
\hline $\mathrm{x}_{3}$ & 0.7 & 0.2 & 0.1 & 0.4900 & 0.0400 & 0.0100 & 24.5294 & 24.6225 & 2.0024 & 2.0100 & 0.5006 & 0.5025 \\
\hline $\mathrm{x}_{4}$ & 0.8 & 0.1 & 0.1 & 0.6400 & 0.0100 & 0.0100 & 23.2640 & 23.4432 & 0.3635 & 0.3663 & 0.3635 & 0.3663 \\
\hline $\mathrm{x}_{5}$ & 0.4 & 0.1 & 0.5 & 0.1600 & 0.0100 & 0.2500 & 7.2848 & 7.0896 & 0.4553 & 0.4431 & 11.3825 & 11.0775 \\
\hline $\mathrm{x}_{6}$ & 0.3 & 0.1 & 0.6 & 0.0900 & 0.0100 & 0.3600 & 4.0734 & 4.3803 & 0.4526 & 0.4867 & 16.2936 & 17.5212 \\
\hline $\mathrm{x}_{7}$ & 0.2 & 0.1 & 0.7 & 0.0400 & 0.0100 & 0.4900 & 1.6080 & 1.7968 & 0.4020 & 0.4492 & 19.6980 & 22.0108 \\
\hline $\mathrm{X}_{8}$ & 0.1 & 0.1 & 0.8 & 0.0100 & 0.0100 & 0.6400 & 0.5118 & 0.5149 & 0.5118 & 0.5149 & 32.7552 & 32.9536 \\
\hline $\mathrm{X}_{9}$ & 0.1 & 0.5 & 0.4 & 0.0100 & 0.2500 & 0.1600 & 0.2362 & 0.2285 & 5.9050 & 5.7125 & 3.7792 & 3.6560 \\
\hline $\mathrm{x}_{10}$ & 0.1 & 0.6 & 0.3 & 0.0100 & 0.3600 & 0.0900 & 0.3644 & 0.3646 & 13.1184 & 13.1256 & 3.2796 & 3.2814 \\
\hline $\mathrm{x}_{11}$ & 0.1 & 0.7 & 0.2 & 0.0100 & 0.4900 & 0.0400 & 0.5386 & 0.5595 & 26.3914 & 27.4155 & 2.1544 & 2.2380 \\
\hline $\mathrm{x}_{12}$ & 0.1 & 0.8 & 0.1 & 0.0100 & 0.6400 & 0.0100 & 0.5882 & 0.6122 & 37.6448 & 39.1808 & 0.5882 & 0.6122 \\
\hline $\mathrm{x}_{13}$ & 0.4 & 0.1 & 0.5 & 0.1600 & 0.0100 & 0.2500 & 8.5712 & 9.6400 & 0.5357 & 0.6025 & 13.3925 & 15.0625 \\
\hline $\mathrm{x}_{14}$ & 0.3 & 0.1 & 0.6 & 0.0900 & 0.0100 & 0.3600 & 5.2083 & 5.4585 & 0.5787 & 0.6065 & 20.8332 & 21.8340 \\
\hline $\mathrm{x}_{15}$ & 0.2 & 0.1 & 0.7 & 0.0400 & 0.0100 & 0.4900 & 2.3924 & 2.4452 & 0.5981 & 0.6113 & 29.3069 & 29.9537 \\
\hline $\mathrm{x}_{16}$ & 0.1 & 0.1 & 0.8 & 0.0100 & 0.0100 & 0.6400 & 0.5295 & 0.5661 & 0.5295 & 0.5661 & 33.8880 & 36.2304 \\
\hline$x_{17}$ & 0.1 & 0.5 & 0.4 & 0.0100 & 0.2500 & 0.1600 & 0.6012 & 0.6214 & 15.0300 & 15.5350 & 9.6192 & 9.9424 \\
\hline $\mathrm{x}_{18}$ & 0.1 & 0.6 & 0.3 & 0.0100 & 0.3600 & 0.0900 & 0.4880 & 0.5171 & 17.5680 & 18.6156 & 4.3920 & 4.6539 \\
\hline $\mathrm{x}_{19}$ & 0.1 & 0.7 & 0.2 & 0.0100 & 0.4900 & 0.0400 & 0.3346 & 0.3561 & 16.3954 & 17.4489 & 1.3384 & 1.4244 \\
\hline $\mathrm{x}_{20}$ & 0.1 & 0.8 & 0.1 & 0.0100 & 0.6400 & 0.0100 & 0.3035 & 0.2940 & 19.4240 & 18.8160 & 0.3035 & 0.2940 \\
\hline $\mathrm{x}_{21}$ & 0.5 & 0.4 & 0.1 & 0.2500 & 0.1600 & 0.0100 & 8.6425 & 8.7675 & 5.5312 & 5.6112 & 0.3457 & 0.3507 \\
\hline $\mathrm{x}_{22}$ & 0.6 & 0.3 & 0.1 & 0.3600 & 0.0900 & 0.0100 & 15.1380 & 15.0732 & 3.7845 & 3.7683 & 0.4205 & 0.4187 \\
\hline $\mathrm{x}_{23}$ & 0.7 & 0.2 & 0.1 & 0.4900 & 0.0400 & 0.0100 & 20.8495 & 20.1880 & 1.7020 & 1.6480 & 0.4255 & 0.4120 \\
\hline $\mathrm{x}_{14}$ & 0.8 & 0.1 & 0.1 & 0.6400 & 0.0100 & 0.0100 & 23.0720 & 17.4528 & 0.3605 & 0.2727 & 0.3605 & 0.2727 \\
\hline $\mathrm{x}_{25}$ & 0.6 & 0.3 & 0.1 & 0.3600 & 0.0900 & 0.0100 & 17.6076 & 17.6580 & 4.4019 & 4.4145 & 0.4891 & 0.4905 \\
\hline $\mathrm{x}_{26}$ & 0.8 & 0.1 & 0.1 & 0.6400 & 0.0100 & 0.0100 & 29.7856 & 31.0848 & 0.4654 & 0.4857 & 0.4654 & 0.4857 \\
\hline $\mathrm{x}_{27}$ & 0.3 & 0.1 & 0.6 & 0.0900 & 0.0100 & 0.3600 & 4.6953 & 4.7169 & 0.5217 & 0.5241 & 18.7812 & 18.8676 \\
\hline $\mathrm{x}_{28}$ & 0.1 & 0.1 & 0.8 & 0.0100 & 0.0100 & 0.6400 & 0.4684 & 0.4707 & 0.4684 & 0.4707 & 29.9776 & 30.1248 \\
\hline $\mathrm{X}_{29}$ & 0.1 & 0.6 & 0.3 & 0.0100 & 0.3600 & 0.0900 & 0.5020 & 0.5551 & 18.0720 & 19.9836 & 4.5180 & 4.9959 \\
\hline $\mathrm{x}_{30}$ & 0.1 & 0.5 & 0.4 & 0.0100 & 0.2500 & 0.1600 & 0.4765 & 0.4941 & 11.9125 & 12.3525 & 7.6240 & 7.9056 \\
\hline $\mathrm{x}_{31}$ & 0.2 & 0.1 & 0.7 & 0.0400 & 0.0100 & 0.4900 & 1.9008 & 2.2288 & 0.4752 & 0.5572 & 23.2848 & 27.3028 \\
\hline $\mathrm{x}_{32}$ & 0.4 & 0.1 & 0.5 & 0.1600 & 0.0100 & 0.2500 & 5.4416 & 5.6176 & 0.3401 & 0.3511 & 8.5025 & 8.7775 \\
\hline $\mathrm{x}_{33}$ & 0.7 & 0.2 & 0.1 & 0.4900 & 0.0400 & 0.0100 & 19.7029 & 20.6388 & 1.6084 & 1.6848 & 0.4021 & 0.4212 \\
\hline $\mathrm{x}_{34}$ & 0.5 & 0.4 & 0.1 & 0.2500 & 0.1600 & 0.0100 & 10.7725 & 10.9875 & 6.8944 & 7.0320 & 0.4309 & 0.4395 \\
\hline \multicolumn{4}{|c|}{$\Sigma\left(\mu^{2}\right)$} & 6.2200 & 5.1100 & 6.1900 & \multicolumn{2}{|c|}{$\Sigma\left(\mu^{2} \cdot \mathrm{x}_{\mathrm{i}}\right)$} & \multicolumn{2}{|c|}{$\Sigma\left(\mu^{2} \cdot \mathrm{x}_{\mathrm{i}}\right)$} & \multicolumn{2}{|c|}{$\Sigma\left(\mu^{2} \cdot \mathrm{x}_{\mathrm{i}}\right)$} \\
\hline \multicolumn{7}{|c|}{ Iteration $1(\mathrm{t}=1)$} & 270.8034 & 270.7107 & 226.7430 & 234.4091 & 300.8864 & 315.9030 \\
\hline \multicolumn{7}{|c|}{$\Sigma\left(\mu^{2} \cdot x_{i}\right) / \Sigma\left(\mu^{2}\right)$} & 43.54 & 43.52 & 44.37 & 45.87 & 48.61 & 51.03 \\
\hline
\end{tabular}

Step 5: Objective Function $\left(\mathrm{P}_{\mathrm{t}}\right)$ using Equation (5) and updating partition matrix $\left(\mu_{\mathrm{ik}}\right)$ using Equation (6), shown on Table (12). Result of calculation $\mathrm{P}_{\mathrm{t}} \rightarrow \mathrm{X}_{\mathrm{ij}} \mathrm{V}_{\mathrm{jk}}, \mathrm{L} \rightarrow\left(\mathrm{X}_{\mathrm{ij}} \cdot \mathrm{V}_{\mathrm{jk}}\right)$ * $\mu^{2}$, Total L $\rightarrow \Sigma\left(X_{\mathrm{ij}} \cdot V_{\mathrm{jk}}\right), \mathrm{LT} \rightarrow\left(\mathrm{X}_{\mathrm{ij}} \cdot \mathrm{V}_{\mathrm{jk}}\right)^{-1 / \mathrm{w}-1}$, Total LT $\left.\rightarrow \Sigma\left(\mathrm{X}_{\mathrm{ij}} \cdot \mathrm{V}_{\mathrm{jk}}\right)^{-1 / \mathrm{w}-1}\right) \rightarrow \mu_{\mathrm{ik}}, \Delta \mathrm{P}_{\mathrm{t}} \rightarrow \mathrm{P}_{\mathrm{t}}-\mathrm{P}_{\mathrm{t}-1}$, shown on Table 12 and Table 13. 
Table 12. Objective Function for Fuzzy C-Means

\begin{tabular}{|c|c|c|c|c|c|c|c|c|c|c|c|}
\hline \multirow{2}{*}{$\frac{\mathrm{x}_{\mathrm{i}}}{\mathrm{x}_{1}}$} & \multicolumn{3}{|c|}{$X_{i j} \cdot V_{k j}$} & \multicolumn{3}{|c|}{$\left(X_{\mathrm{ij}} \cdot \mathrm{V}_{\mathrm{kj}}\right) * \mu^{2}$} & \multirow{2}{*}{$\begin{array}{r}\begin{array}{c}\Sigma\left(\mathrm{X}_{\mathrm{ij}} \cdot \mathrm{V}_{\mathrm{kj}}\right) \\
* \mu^{2}\end{array} \\
24.3685\end{array}$} & \multicolumn{3}{|c|}{$\left(X_{\mathrm{ij}} \cdot \mathrm{V}_{\mathrm{kj}}\right)^{1 / \mathrm{w}-1}$} & \multirow{2}{*}{$\begin{array}{c}\begin{array}{c}\Sigma\left(\mathrm{X}_{\mathrm{ij}} \cdot \mathrm{V}_{\mathrm{kj}}\right. \\
1 / \mathrm{w}-1\end{array} \\
3.705717\end{array}$} \\
\hline & 73.0712 & 38.1126 & 0.2728 & 18.2678 & 6.0980 & 0.0027 & & 0.0137 & 0.0262 & 3.6658 & \\
\hline $\mathrm{x}_{2}$ & 117.6868 & 73.4585 & 4.5441 & 42.3672 & 6.6113 & 0.0454 & 49.0240 & 0.0085 & 0.0136 & 0.2201 & 0.242174 \\
\hline $\mathrm{x}_{3}$ & 87.8003 & 51.5101 & 2.7223 & 43.0222 & 2.0604 & 0.0272 & 45.1098 & 0.0114 & 0.0194 & 0.3673 & 0.398146 \\
\hline $\mathrm{x}_{4}$ & 99.1687 & 149.7851 & 357.7570 & 63.4680 & 1.4979 & 3.5776 & 68.5434 & 0.0101 & 0.0067 & 0.0028 & 0.019555 \\
\hline $\mathrm{x}_{5}$ & 4.5899 & 3.7818 & 54.6946 & 0.7344 & 0.0378 & 13.6737 & 14.4459 & 0.2179 & 0.2644 & 0.0183 & 0.500575 \\
\hline $\mathrm{x}_{6}$ & 29.4624 & 8.6131 & 16.8027 & 2.6516 & 0.0861 & 6.0490 & 8.7867 & 0.0339 & 0.1161 & 0.0595 & 0.209558 \\
\hline $\mathrm{x}_{7}$ & 13.0917 & 18.3165 & 108.0883 & 0.5237 & 0.1832 & 52.9633 & 53.6701 & 0.0764 & 0.0546 & 0.0093 & 0.140231 \\
\hline $\mathrm{x}_{8}$ & 121.8866 & 77.8983 & 6.8204 & 1.2189 & 0.7790 & 4.3650 & 6.3629 & 0.0082 & 0.0128 & 0.1466 & 0.167662 \\
\hline $\mathrm{X}_{9}$ & 824.0650 & 960.7035 & 1418.7844 & 8.2407 & 240.1759 & 227.0055 & 475.4220 & 0.0012 & 0.0010 & 0.0007 & 0.002959 \\
\hline $\mathrm{x}_{10}$ & 100.2555 & 151.5205 & 360.4850 & 1.0026 & 54.5474 & 32.4436 & 87.9936 & 0.0100 & 0.0066 & 0.0028 & 0.019348 \\
\hline $\mathrm{x}_{11}$ & 260.9933 & 191.5680 & 51.7416 & 2.6099 & 93.8683 & 2.0697 & 98.5479 & 0.0038 & 0.0052 & 0.0193 & 0.028378 \\
\hline $\mathrm{x}_{12}$ & 546.7513 & 444.2749 & 208.0217 & 5.4675 & 284.3360 & 2.0802 & 291.8837 & 0.0018 & 0.0023 & 0.0048 & 0.008887 \\
\hline $\mathrm{x}_{13}$ & 380.4558 & 291.3047 & 109.5439 & 60.8729 & 2.9130 & 27.3860 & 91.1720 & 0.0026 & 0.0034 & 0.0091 & 0.015190 \\
\hline $\mathrm{x}_{14}$ & 498.7670 & 400.5559 & 178.2356 & 44.8890 & 4.0056 & 64.1648 & 113.0594 & 0.0020 & 0.0025 & 0.0056 & 0.010112 \\
\hline $\mathrm{x}_{15}$ & 574.8133 & 471.1069 & 227.3953 & 22.9925 & 4.7111 & 111.4237 & 139.1273 & 0.0017 & 0.0021 & 0.0044 & 0.008260 \\
\hline $\mathrm{x}_{16}$ & 259.8742 & 188.8664 & 49.9361 & 2.5987 & 1.8887 & 31.9591 & 36.4465 & 0.0038 & 0.0053 & 0.0200 & 0.029168 \\
\hline $\mathrm{x}_{17}$ & 621.5853 & 512.6143 & 255.8496 & 6.2159 & 128.1536 & 40.9359 & 175.3053 & 0.0016 & 0.0020 & 0.0039 & 0.007468 \\
\hline $\mathrm{x}_{18}$ & 94.7268 & 53.6786 & 0.4931 & 0.9473 & 19.3243 & 0.0444 & 20.3159 & 0.0106 & 0.0186 & 2.0280 & 2.057144 \\
\hline $\mathrm{x}_{19}$ & 164.1661 & 224.4020 & 467.3884 & 1.6417 & 109.9570 & 18.6955 & 130.2942 & 0.0061 & 0.0045 & 0.0021 & 0.012687 \\
\hline $\mathrm{x}_{20}$ & 373.3592 & 467.9752 & 801.4193 & 3.7336 & 299.5041 & 8.0142 & 311.2519 & 0.0027 & 0.0021 & 0.0012 & 0.006063 \\
\hline $\mathrm{x}_{21}$ & 151.8633 & 212.7838 & 451.9409 & 37.9658 & 34.0454 & 4.5194 & 76.5306 & 0.0066 & 0.0047 & 0.0022 & 0.013497 \\
\hline $\mathrm{x}_{22}$ & 4.9439 & 21.4146 & 126.9999 & 1.7798 & 1.9273 & 1.2700 & 4.9771 & 0.2023 & 0.0467 & 0.0079 & 0.256841 \\
\hline $\mathrm{x}_{23}$ & 6.3698 & 25.1546 & 133.4206 & 3.1212 & 1.0062 & 1.3342 & 5.4616 & 0.1570 & 0.0398 & 0.0075 & 0.204241 \\
\hline $\mathrm{x}_{24}$ & 320.2107 & 415.3200 & 722.4622 & 204.9348 & 4.1532 & 7.2246 & 216.3127 & 0.0031 & 0.0024 & 0.0014 & 0.006915 \\
\hline $\mathrm{x}_{25}$ & 59.4154 & 30.6855 & 4.0288 & 21.3896 & 2.7617 & 0.0403 & 24.1915 & 0.0168 & 0.0326 & 0.2482 & 0.297632 \\
\hline $\mathrm{x}_{26}$ & 34.4909 & 11.9743 & 10.3519 & 22.0742 & 0.1197 & 0.1035 & 22.2974 & 0.0290 & 0.0835 & 0.0966 & 0.209106 \\
\hline $\mathrm{x}_{27}$ & 153.5052 & 103.5398 & 14.5768 & 13.8155 & 1.0354 & 5.2476 & 20.0985 & 0.0065 & 0.0097 & 0.0686 & 0.084775 \\
\hline $\mathrm{x}_{28}$ & 23.4902 & 7.5227 & 18.8440 & 0.2349 & 0.0752 & 12.0602 & 12.3703 & 0.0426 & 0.1329 & 0.0531 & 0.228569 \\
\hline $\mathrm{x}_{29}$ & 188.0859 & 126.8399 & 22.5639 & 1.8809 & 45.6624 & 2.0307 & 49.5740 & 0.0053 & 0.0079 & 0.0443 & 0.057519 \\
\hline $\mathrm{x}_{30}$ & 51.5737 & 23.2557 & 3.5574 & 0.5157 & 5.8139 & 0.5692 & 6.8988 & 0.0194 & 0.0430 & 0.2811 & 0.343497 \\
\hline $\mathrm{x}_{31}$ & 164.6362 & 106.8782 & 23.1395 & 6.5854 & 1.0688 & 11.3384 & 18.9926 & 0.0061 & 0.0094 & 0.0432 & 0.058647 \\
\hline$x_{32}$ & 161.5459 & 223.2135 & 466.7020 & 25.8473 & 2.2321 & 116.6755 & 144.7550 & 0.0062 & 0.0045 & 0.0021 & 0.012813 \\
\hline $\mathrm{x}_{33}$ & 13.0398 & 31.4078 & 150.0009 & 6.3895 & 1.2563 & 1.5000 & 9.1458 & 0.0767 & 0.0318 & 0.0067 & 0.115194 \\
\hline $\mathrm{x}_{34}$ & 0.3829 & 5.3410 & 80.6423 & 0.0957 & 0.8546 & 0.8064 & 1.7567 & 2.6114 & 0.1872 & 0.0124 & 2.811066 \\
\hline \multirow{3}{*}{\multicolumn{3}{|c|}{ Centroid }} & 43.54 & 43.52 & \multicolumn{2}{|c|}{ Obj. function $\left(\mathrm{P}_{\mathrm{t}}\right)$} & 2854.4937 & \multirow{3}{*}{\multicolumn{4}{|c|}{ If $\Delta\left(\mathrm{P}_{\mathrm{t}}\right)>(\varepsilon)$, then next iteration }} \\
\hline & & & 44.37 & 45.87 & \multicolumn{2}{|c|}{$\Delta\left(\mathrm{P}_{\mathrm{t}}\right)$} & 2854.4937 & & & & \\
\hline & & & 48.61 & 51.03 & \multicolumn{2}{|c|}{ Min. error $(\varepsilon)$} & 0.000001 & & & & \\
\hline
\end{tabular}

Step 6: Iteration $(\mathrm{t}=1)$ until maximum iteration $(\mathrm{t}=100)$. If $\Delta\left(\mathrm{P}_{\mathrm{t}}\right)>(\varepsilon)$ or $\mathrm{t}<\max$-iter, then back to Step 4. On iteration 43, $\Delta\left(\mathrm{P}_{\mathrm{t}}\right)<(\varepsilon)$, stop iteration, next to Step 7.

Step 7: Grouping max. value, min. value, count of data point to new centroid based on max. value of data point in each new centroid become cluster, can be seen on Table 14 . 
Table 13. Calculation Update Partition Matrix for Fuzzy C-Means

\begin{tabular}{|l|l|l|l|}
\hline \multicolumn{1}{|c|}{ Matrix $(\mu)$} & \multicolumn{1}{|c|}{ Excel } & \multicolumn{1}{c|}{ Matrix $(\mu)$} & \multicolumn{1}{c|}{ Excel } \\
\hline $\mathrm{X}_{\mathrm{ij}} \cdot \mathrm{V}_{\mathrm{kj}}$ & $\begin{array}{l}=((\mathrm{C} 7-\$ \mathrm{~B} \$ 84) *(\mathrm{C} 7-\$ \mathrm{~B} \$ 84))+ \\
((\mathrm{D} 7-\$ \mathrm{C} \$ 84) *(\mathrm{D} 7-\$ \mathrm{C} \$ 84))\end{array}$ & $\Sigma\left(\mathrm{X}_{\mathrm{ij} \cdot} \cdot \mathrm{V}_{\mathrm{kj}}\right)^{-1 / \mathrm{w}-1}$ & $=\mathrm{SUM}(\mathrm{O} 84: \mathrm{Q} 84)$ \\
\hline $\mathrm{X}_{\mathrm{ij}} \cdot \mathrm{V}_{\mathrm{kj}} * \mu^{2}$ & $=\mathrm{E} 84 * \mathrm{~F} 46$ & $\mathrm{P}_{\mathrm{t}}$ & $=\mathrm{SUM}(\mathrm{M} 84: \mathrm{M} 117)$ \\
\hline$\Sigma\left(\mathrm{X}_{\mathrm{ij} \cdot} \cdot \mathrm{V}_{\mathrm{kj}}\right) * \mu^{2}$ & $=\mathrm{SUM}(\mathrm{I} 84: \mathrm{K} 84)$ & $\Delta \mathrm{P}_{\mathrm{t}} \rightarrow \mathrm{P}_{\mathrm{t}}-\mathrm{P}_{\mathrm{t}-1}$, & $=\mathrm{ABS}(\mathrm{B} 89-0)$ \\
\hline$\left(\mathrm{X}_{\mathrm{ij} \cdot} \mathrm{V}_{\mathrm{kj}}\right)^{-1 / \mathrm{w}-1}$ & $=\mathrm{POWER}(\mathrm{E} 84,(-1 /(\$ \mathrm{H} \$ 7-1)))$ & Cluster $\left(\mathrm{C}_{\mathrm{i}}\right)$ & $=\mathrm{MAX}(\mathrm{J} 3297: \mathrm{Q} 3297)$ \\
\hline
\end{tabular}

Table 14. Membership for Fuzzy C-Means

\begin{tabular}{|c|c|c|c|c|c|c|c|c|}
\hline $\mathrm{x}_{\mathrm{i}}$ & & Iember & & $\max$ & $\mathrm{C}_{\mathrm{i}}$ & $\mathrm{C}_{1}$ & $\mathrm{C}_{2}$ & $\mathrm{C}_{3}$ \\
\hline $\mathrm{x}_{1}$ & 0.0127 & 0.9383 & 0.0490 & 0.9383 & $\mathrm{C}_{2}$ & & Aceh & \\
\hline$x_{2}$ & 0.0300 & 0.7468 & 0.2232 & 0.7468 & $\mathrm{C}_{2}$ & & Sumatera Utara & \\
\hline $\mathrm{x}_{3}$ & 0.0213 & 0.8762 & 0.1025 & 0.8762 & $\mathrm{C}_{2}$ & & Sumatera Barat & \\
\hline $\mathrm{x}_{4}$ & 0.9202 & 0.0623 & 0.0175 & 0.9202 & $\mathrm{C}_{1}$ & Riau & & \\
\hline $\mathrm{x}_{5}$ & 0.0645 & 0.8853 & 0.0502 & 0.8853 & $\mathrm{C}_{2}$ & & Jambi & \\
\hline $\mathrm{x}_{6}$ & 0.0076 & 0.9802 & 0.0123 & 0.9802 & $\mathrm{C}_{2}$ & & Sumatera Selatan & \\
\hline $\mathrm{x}_{7}$ & 0.2257 & 0.6872 & 0.0871 & 0.6872 & $\mathrm{C}_{2}$ & & Bengkulu & \\
\hline $\mathrm{x}_{8}$ & 0.0319 & 0.7219 & 0.2463 & 0.7219 & $\mathrm{C}_{2}$ & & Lampung & \\
\hline $\mathrm{x}_{9}$ & 0.7866 & 0.1417 & 0.0717 & 0.7866 & $\mathrm{C}_{1}$ & Bangka Belitung & & \\
\hline $\mathrm{x}_{10}$ & 0.9236 & 0.0596 & 0.0168 & 0.9236 & $\mathrm{C}_{1}$ & Kepulauan Riau & & \\
\hline $\mathrm{x}_{11}$ & 0.0139 & 0.1199 & 0.8662 & 0.8662 & $\mathrm{C}_{3}$ & & & DKI Jakarta \\
\hline $\mathrm{x}_{12}$ & 0.0084 & 0.0386 & 0.9530 & 0.9530 & $\mathrm{C}_{3}$ & & & Jawa Barat \\
\hline $\mathrm{x}_{13}$ & 0.0062 & 0.0375 & 0.9563 & 0.9563 & $\mathrm{C}_{3}$ & & & Jawa Tengah \\
\hline $\mathrm{x}_{14}$ & 0.0043 & 0.0211 & 0.9745 & 0.9745 & $\mathrm{C}_{3}$ & & & DI Yogyakarta \\
\hline $\mathrm{x}_{15}$ & 0.0120 & 0.0529 & 0.9351 & 0.9351 & $\mathrm{C}_{3}$ & & & Jawa Timur \\
\hline $\mathrm{x}_{16}$ & 0.0147 & 0.1285 & 0.8568 & 0.8568 & $\mathrm{C}_{3}$ & & & Banten \\
\hline $\mathrm{x}_{17}$ & 0.0158 & 0.0661 & 0.9181 & 0.9181 & $\mathrm{C}_{3}$ & & & Bali \\
\hline $\mathrm{x}_{18}$ & 0.0223 & 0.8606 & 0.1171 & 0.8606 & $\mathrm{C}_{2}$ & & Nusa Tenggara Barat & \\
\hline $\mathrm{x}_{19}$ & 0.9796 & 0.0154 & 0.0050 & 0.9796 & $\mathrm{C}_{1}$ & Nusa Tenggara Timur & & \\
\hline $\mathrm{x}_{20}$ & 0.9414 & 0.0415 & 0.0171 & 0.9414 & $\mathrm{C}_{1}$ & Kalimantan Barat & & \\
\hline $\mathrm{x}_{21}$ & 0.9862 & 0.0105 & 0.0033 & 0.9862 & $\mathrm{C}_{1}$ & Kalimantan Tengah & & \\
\hline $\mathrm{x}_{22}$ & 0.2953 & 0.6210 & 0.0837 & 0.6210 & $\mathrm{C}_{2}$ & & Kalimantan Selatan & \\
\hline $\mathrm{x}_{23}$ & 0.3122 & 0.6027 & 0.0851 & 0.6027 & $\mathrm{C}_{2}$ & & Kalimantan Timur & \\
\hline $\mathrm{x}_{24}$ & 0.9072 & 0.0663 & 0.0265 & 0.9072 & $\mathrm{C}_{1}$ & Kalimantan Utara & & \\
\hline $\mathrm{x}_{25}$ & 0.0090 & 0.9630 & 0.0280 & 0.9630 & $\mathrm{C}_{2}$ & & Sulawesi Utara & \\
\hline $\mathrm{x}_{26}$ & 0.0004 & 0.9987 & 0.0008 & 0.9987 & $\mathrm{C}_{2}$ & & Sulawesi Tengah & \\
\hline $\mathrm{x}_{27}$ & 0.0349 & 0.5567 & 0.4084 & 0.5567 & $\mathrm{C}_{2}$ & & Sulawesi Selatan & \\
\hline $\mathrm{x}_{28}$ & 0.0049 & 0.9874 & 0.0076 & 0.9874 & $\mathrm{C}_{2}$ & & Sulawesi Tenggara & \\
\hline $\mathrm{x}_{29}$ & 0.0338 & 0.4173 & 0.5489 & 0.5489 & $\mathrm{C}_{3}$ & & & Gorontalo \\
\hline $\mathrm{x}_{30}$ & 0.0034 & 0.9871 & 0.0094 & 0.9871 & $\mathrm{C}_{2}$ & & Sulawesi Barat & \\
\hline $\mathrm{x}_{31}$ & 0.0455 & 0.5797 & 0.3747 & 0.5797 & $\mathrm{C}_{2}$ & & Maluku & \\
\hline $\mathrm{x}_{32}$ & 0.9878 & 0.0092 & 0.0030 & 0.9878 & $\mathrm{C}_{1}$ & Maluku Utara & & \\
\hline $\mathrm{x}_{33}$ & 0.3837 & 0.5328 & 0.0836 & 0.5328 & $\mathrm{C}_{2}$ & & Papua Barat & \\
\hline $\mathrm{x}_{34}$ & 0.1383 & 0.7929 & 0.0688 & 0.7929 & $\mathrm{C}_{2}$ & & Papua & \\
\hline
\end{tabular}




\subsection{Comparison}

Comparison of member clustering in K-Means and Fuzzy C-Means shown on Table 15.

Table 15. Comparison of Membership Clustering

\begin{tabular}{|c|c|c|c|c|c|}
\hline \multicolumn{3}{|c|}{ Membership K-Means Clustering } & \multicolumn{3}{|c|}{ Membership Fuzzy C-Means Clustering } \\
\hline $\mathrm{C}_{1}$ & $\mathrm{C}_{2}$ & $\mathrm{C}_{3}$ & $\mathrm{C}_{1}$ & $\mathrm{C}_{2}$ & $\mathrm{C}_{3}$ \\
\hline $\mathrm{x}_{4}$ & $\mathrm{x}_{1}$ & $\mathrm{x}_{11}$ & $\mathrm{x}_{4}$ & $\mathrm{x}_{1}$ & $\mathrm{x}_{11}$ \\
\hline $\mathrm{X}_{9}$ & $\mathrm{x}_{2}$ & $\mathrm{x}_{12}$ & $\mathrm{X}_{9}$ & $\mathrm{x}_{2}$ & $x_{12}$ \\
\hline $\mathrm{x}_{10}$ & $x_{3}$ & $\mathrm{x}_{13}$ & $\mathrm{x}_{10}$ & $\mathrm{x}_{3}$ & $x_{13}$ \\
\hline $\mathrm{x}_{19}$ & $\mathrm{x}_{5}$ & $\mathrm{x}_{14}$ & $\mathrm{X}_{19}$ & $\mathrm{x}_{5}$ & $\mathrm{x}_{14}$ \\
\hline $\mathrm{X}_{20}$ & $\mathrm{x}_{6}$ & $\mathrm{x}_{15}$ & $\mathrm{x}_{20}$ & $\mathrm{x}_{6}$ & $\mathrm{x}_{15}$ \\
\hline $\mathrm{x}_{21}$ & $\mathrm{x}_{7}$ & $\mathrm{x}_{16}$ & $\mathrm{x}_{21}$ & $\mathrm{x}_{7}$ & $\mathrm{x}_{16}$ \\
\hline $\mathrm{x}_{22}$ & $\mathrm{x}_{8}$ & $x_{17}$ & $\mathrm{x}_{24}$ & $\mathrm{X}_{8}$ & $x_{17}$ \\
\hline $\mathrm{x}_{23}$ & $\mathrm{x}_{18}$ & & $x_{32}$ & $\mathrm{x}_{18}$ & $\mathrm{X}_{29}$ \\
\hline $\mathrm{X}_{24}$ & $\mathrm{X}_{25}$ & & & $x_{22}$ & \\
\hline $\mathrm{x}_{32}$ & $\mathrm{x}_{26}$ & & & $\mathrm{x}_{23}$ & \\
\hline \multirow[t]{8}{*}{$x_{33}$} & $\mathrm{X}_{27}$ & & & $\mathrm{X}_{25}$ & \\
\hline & $\mathrm{X}_{28}$ & & & $\mathrm{x}_{26}$ & \\
\hline & $\mathrm{x}_{29}$ & & & $x_{27}$ & \\
\hline & $\mathrm{x}_{30}$ & & & $\mathrm{X}_{28}$ & \\
\hline & $\mathrm{x}_{31}$ & & & $\mathrm{x}_{30}$ & \\
\hline & $\mathrm{x}_{34}$ & & & $\mathrm{x}_{31}$ & \\
\hline & & & & $\mathrm{x}_{33}$ & \\
\hline & & & & $x_{34}$ & \\
\hline $\mathrm{N}=11$ & $\mathrm{~N}=16$ & $\mathrm{~N}=7$ & $\mathrm{~N}=8$ & $\mathrm{~N}=18$ & $\mathrm{~N}=8$ \\
\hline
\end{tabular}

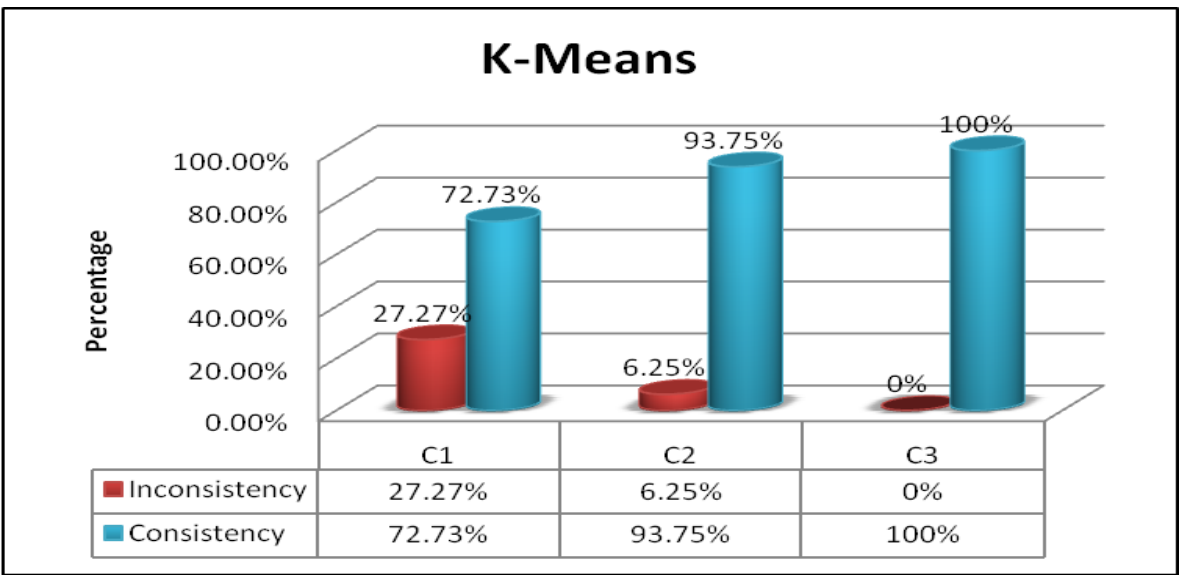

Figure 4. Comparison of Consistency K-Means

Figure 4, shown comparison of membership in K-Means, on Cluster $1\left(\mathrm{C}_{1}\right)$, data point $(\mathrm{N}=11), 3$ data point $\left(\mathrm{x}_{22}, \mathrm{x}_{23}, \mathrm{x}_{33}\right)$ inconsistency $\rightarrow 3 / 11 * 100 \%=27.27 \%$ and consistency $\rightarrow 8 / 11 * 100 \%=72.73 \%$. Cluster $2\left(\mathrm{C}_{2}\right)$, point data $(\mathrm{N}=16), 1$ data point $\left(\mathrm{x}_{29}\right)$ inconsistency $\rightarrow 1 / 16^{*} 100 \%=6.25 \%$ and consistency $\rightarrow 15 / 16 * 100 \%=93.75 \%$. Cluster 3 $\left(\mathrm{C}_{3}\right)$, data point $(\mathrm{N}=7), 0 \%$ inconsistency and $100 \%$ consistency. 


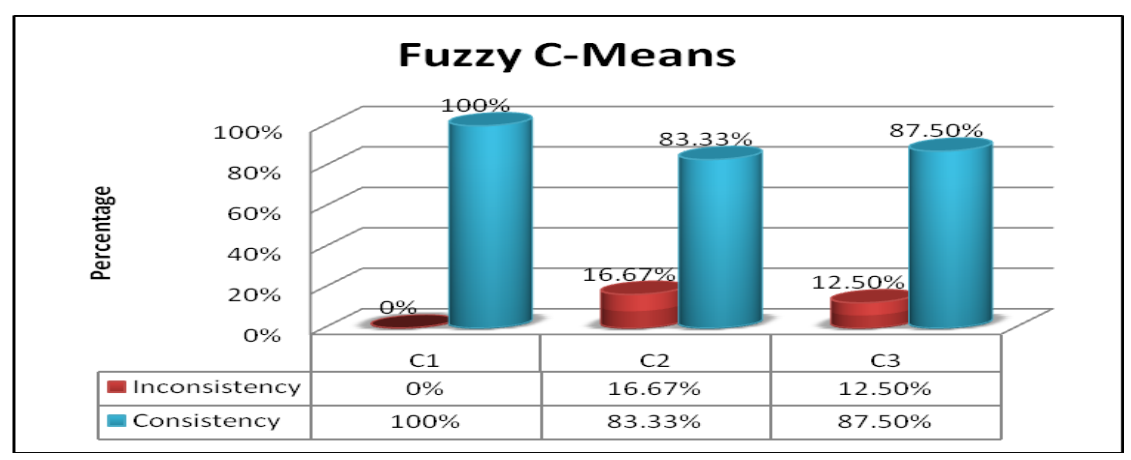

Figure 5. Comparison of Fuzzy C-Means

Figure 5, shown comparison of membership in Fuzzy C-Means, on Cluster $1\left(\mathrm{C}_{1}\right)$, data point $(\mathrm{N}=8), 0 \%$ inconsistency and $100 \%$ consistency. Cluster $2\left(\mathrm{C}_{2}\right)$, data point $(\mathrm{N}=18), 3$ data point $\left(\mathrm{x}_{22}, \mathrm{x}_{23}, \mathrm{x}_{33}\right)$ inconsistency $\rightarrow 3 / 18 * 100 \%=16.67 \%$, and consistency $\rightarrow$ $15 / 18 * 100 \%=83.33 \%$. Cluster $3\left(\mathrm{C}_{3}\right), 1$ data point $\left(\mathrm{x}_{29}\right)$ inconsistency $\rightarrow 1 / 8 * 100 \%=$ $12.50 \%$, and consistency $\rightarrow 7 / 8 * 100 \%=87.50 \%$.

Table 16. Comparison of Rice Productivity for K-Means Clustering

\begin{tabular}{|c|c|c|c|c|c|c|c|c|c|c|c|}
\hline $\mathrm{x}_{\mathrm{i}}$ & $\mathrm{C}_{1}$ & $\begin{array}{c}2014 \\
(\mathrm{Ku} / \mathrm{Ha}) \\
\end{array}$ & $\begin{array}{c}2015 \\
(\mathrm{Ku} / \mathrm{Ha}) \\
\end{array}$ & $\mathrm{x}_{\mathrm{i}}$ & $\mathrm{C}_{2}$ & $\begin{array}{c}2014 \\
(\mathrm{Ku} / \mathrm{Ha}) \\
\end{array}$ & $\begin{array}{c}2015 \\
(\mathrm{Ku} / \mathrm{Ha}) \\
\end{array}$ & $\mathrm{x}_{\mathrm{i}}$ & $\mathrm{C}_{3}$ & $\begin{array}{c}2014 \\
(\mathrm{Ku} / \mathrm{Ha}) \\
\end{array}$ & $\begin{array}{c}2015 \\
(\mathrm{Ku} / \mathrm{Ha}) \\
\end{array}$ \\
\hline $\mathrm{X}_{9}$ & BangkaBelitung & 23,62 & 22,85 & $\mathrm{x}_{2}$ & SumateraUtara & 50,62 & 51,74 & $\mathrm{X}_{12}$ & Jawa Barat & 58,82 & 61,22 \\
\hline $\mathrm{x}_{19}$ & NTT & 33,46 & 35,61 & $\mathrm{X}_{5}$ & Jambi & 45,53 & 44,31 & $\mathrm{X}_{14}$ & DIYogyakarta & 57,87 & 60,65 \\
\hline $\mathrm{X}_{20}$ & KalimantanBarat & 30,35 & 29,40 & $\mathrm{x}_{6}$ & SumateraSelatan & 45,26 & 48,67 & $\mathrm{x}_{15}$ & Jawa Timur & 59,81 & 61,13 \\
\hline $\mathrm{x}_{21}$ & KalimantanTengah & 34,57 & 35,07 & $\mathrm{x}_{7}$ & Bengkulu & 40,20 & 44,92 & $\mathrm{x}_{16}$ & Banten & 52,95 & 56,61 \\
\hline $\mathrm{X}_{24}$ & Kalimantan Utara & 36,05 & 27,27 & $\mathrm{X}_{25}$ & Sulawesi Utara & 48,91 & 49,05 & \multirow{6}{*}{\multicolumn{4}{|c|}{$\begin{array}{c}\text { Rice Productivity in } \mathrm{C}_{3}>\text { Indonesia } \\
=\text { increase }\end{array}$}} \\
\hline $\mathrm{X}_{32}$ & Maluku Utara & 34,01 & 35,11 & $\mathrm{X}_{26}$ & SulawesiTengah & 46,54 & 48,57 & & & & \\
\hline $\mathrm{X}_{33}$ & Papua Barat & 40,21 & 42,12 & $\mathrm{X}_{27}$ & SulawesiSelatan & 52,17 & 52,41 & & & & \\
\hline \multirow{3}{*}{\multicolumn{4}{|c|}{$\begin{array}{c}\text { Rice Productivity in } \mathrm{C}_{1}<\text { Indonesia } \\
=\text { decrease }\end{array}$}} & $\mathrm{X}_{28}$ & SulawesiTenggara & 46,84 & 47,07 & & & & \\
\hline & & & & $\mathrm{X}_{29}$ & Gorontalo & 50,20 & 55,51 & & & & \\
\hline & & & & $\mathrm{x}_{30}$ & Sulawesi Barat & 47,65 & 49,41 & & & & \\
\hline \multicolumn{2}{|c|}{ Indonesia } & 51,35 & 53,41 & \multicolumn{2}{|c|}{ Indonesia } & 51,35 & 53,41 & \multicolumn{2}{|c|}{ Indonesia } & 51,35 & 53,41 \\
\hline
\end{tabular}

Table 17. Comparison of Rice Productivity for Fuzzy C-Means Clustering

\begin{tabular}{|c|c|c|c|c|c|c|c|c|c|c|c|}
\hline $\mathrm{x}_{\mathrm{i}}$ & $\mathrm{C}_{1}$ & $\begin{array}{c}2014 \\
(\mathrm{Ku} / \mathrm{Ha})\end{array}$ & $\begin{array}{c}2015 \\
(\mathrm{Ku} / \mathrm{Ha}) \\
\end{array}$ & $\mathrm{x}_{\mathrm{i}}$ & $\mathrm{C}_{2}$ & $\begin{array}{c}2014 \\
(\mathrm{Ku} / \mathrm{Ha}) \\
\end{array}$ & $\begin{array}{c}2015 \\
(\mathrm{Ku} / \mathrm{Ha}) \\
\end{array}$ & $\mathrm{x}_{\mathrm{i}}$ & $\mathrm{C}_{3}$ & $\begin{array}{c}2014 \\
(\mathrm{Ku} / \mathrm{Ha})\end{array}$ & $\begin{array}{c}2015 \\
(\mathrm{Ku} / \mathrm{Ha}) \\
\end{array}$ \\
\hline $\mathrm{X}_{9}$ & BangkaBelitung & 23,62 & 22,85 & $\mathrm{X}_{2}$ & SumateraUtara & 50,62 & 51,74 & $\mathrm{x}_{12}$ & Jawa Barat & 58,82 & 61,22 \\
\hline $\mathrm{x}_{19}$ & NTT & 33,46 & 35,61 & $\mathrm{X}_{5}$ & Jambi & 45,53 & 44,31 & $\mathrm{x}_{14}$ & DIYogyakarta & 57,87 & 60,65 \\
\hline $\mathrm{X}_{20}$ & KalimantanBarat & 30,35 & 29,40 & $\mathrm{x}_{6}$ & SumateraSelatan & 45,26 & 48,67 & $\mathrm{X}_{15}$ & Jawa Timur & 59,81 & 61,13 \\
\hline $\mathrm{X}_{21}$ & KalimantanTengah & 34,57 & 35,07 & $\mathrm{X}_{7}$ & Bengkulu & 40,20 & 44,92 & $\mathrm{x}_{16}$ & Banten & 52,95 & 56,61 \\
\hline \multirow{7}{*}{\multicolumn{4}{|c|}{$\begin{array}{c}\text { Rice Productivity in } \mathrm{C}_{1}<\text { Indonesia } \\
=\text { decrease }\end{array}$}} & $\mathrm{x}_{22}$ & KalimantanSelatan & 42,05 & 41,87 & \multirow{7}{*}{\multicolumn{4}{|c|}{$\begin{array}{c}\text { Rice Productivity in } \mathrm{C}_{3}>\text { Indonesia } \\
=\text { increase }\end{array}$}} \\
\hline & & & & $\mathrm{x}_{23}$ & Kalimantan Timur & 42,55 & 41,20 & & & & \\
\hline & & & & $\mathrm{x}_{25}$ & Sulawesi Utara & 48,91 & 49,05 & & & & \\
\hline & & & & $\mathrm{X}_{26}$ & Sulawesi Tengah & 46,54 & 48,57 & & & & \\
\hline & & & & $\mathrm{X}_{27}$ & Sulawesi Selatan & 52,17 & 52,41 & & & & \\
\hline & & & & $\mathrm{X}_{28}$ & SulawesiTenggara & 46,84 & 47,07 & & & & \\
\hline & & & & \multicolumn{4}{|c|}{$\begin{array}{c}\text { Rice Productivity in } \mathrm{C}_{2}<\text { Indonesia } \\
=\text { decrease }\end{array}$} & & & & \\
\hline \multicolumn{2}{|c|}{ Indonesia } & 51,35 & 53,41 & Ind & hesia & 51,35 & 53,41 & \multicolumn{2}{|c|}{ Indonesia } & 51,35 & 53,41 \\
\hline
\end{tabular}


Table 16, shown Comparison of Rice Productivity $\mathrm{C}_{1}$ and Productivity of National Food (Indonesia) is decrease. Comparison of Rice Productivity $\mathrm{C}_{2}$ and Productivity of National Food (Indonesia) is decrease. Comparison of Rice Productivity $\mathrm{C}_{3}$ and Productivity of National Food (Indonesia) is increase. Cluster $2\left(\mathrm{C}_{2}\right)$, there is 3 data point data $\left(\mathrm{x}_{27}, \mathrm{x}_{29}, \mathrm{x}_{31}\right): 3 / 16 * 100 \%=18.75 \% \rightarrow$ anomaly.

Table 17, shown Comparison of Rice Productivity $\mathrm{C}_{1}$ and Productivity of National Food (Indonesia) is decrease. Comparison of Rice Productivity $\mathrm{C}_{2}$ and Productivity of National Food (Indonesia) is decrease. Comparison of Rice Productivity $\mathrm{C}_{3}$ and Productivity of National Food (Indonesia) is increase. Cluster $2\left(\mathrm{C}_{2}\right)$, there is 2 data point data $\left(\mathrm{x}_{27}, \mathrm{x}_{31}\right): 2 / 18 * 100 \%=11.11 \% \rightarrow$ anomaly. Cluster $2\left(\mathrm{C}_{3}\right)$, there is 1 data point data $\left(\mathrm{x}_{29}\right): 1 / 8 * 100 \%=12.50 \% \rightarrow$ anomaly.

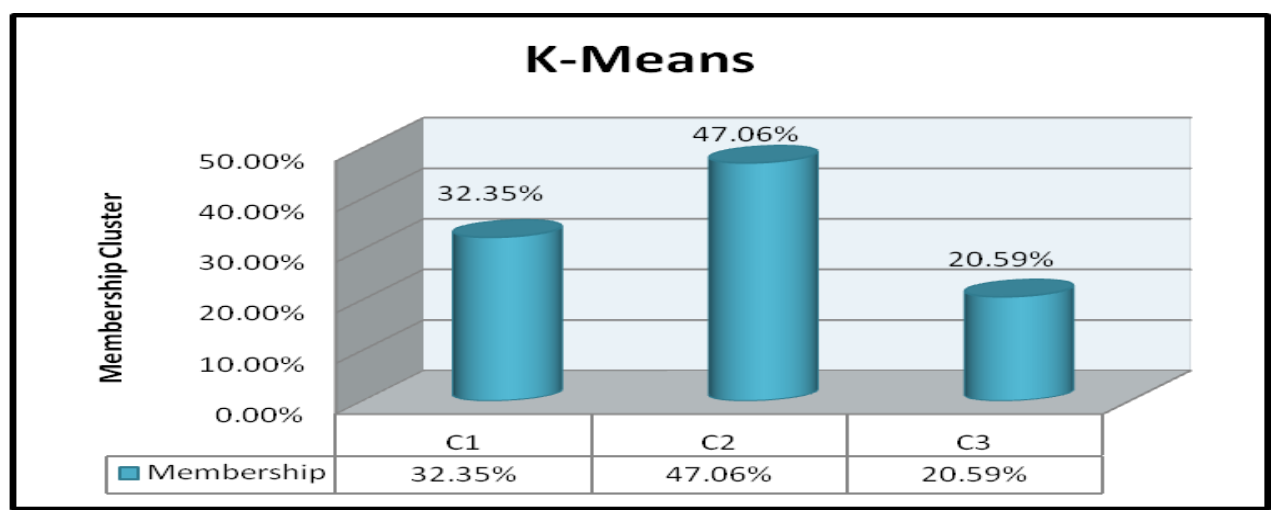

Figure 6. Membership Cluster of K-Means

Figure 6, shown membership cluster in K-Means Clustering. Percentage membership data point in Cluster 1 is 11 from 34 data point using $=(11 / 34) * 100 \%=32.35 \%$. Percentage membership data point in Cluster 2 is 16 from 34 data point using = $(16 / 34) * 100 \%=47.06 \%$. Percentage membership data point in Cluster 3 is 7 from 34 data point using $=(7 / 34) * 100 \%=20.59 \%$.

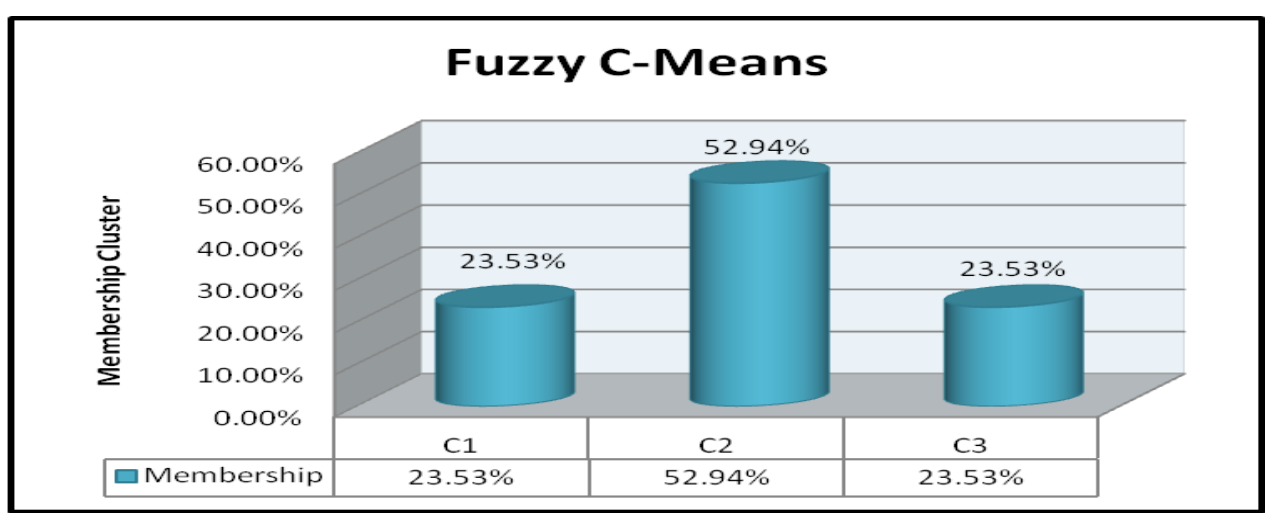

Figure 7. Membership Cluster of Fuzzy C-Means

Figure 7, shown membership cluster in Fuzzy C-Means Clustering. Percentage membership data point in Cluster 1 is 8 from 34 data point using $=(8 / 34) * 100 \%=$ $23.53 \%$. Percentage membership data point in Cluster 2 is 18 from 34 data point using $=$ $(18 / 34) * 100 \%=52.94 \%$. Percentage membership data point in Cluster 3 is 8 from 34 data point using $=(8 / 34) * 100 \%=23.53 \%$.

\section{Conclusion}


Experimental result of clustering using K-Means and Fuzzy C-Means produce three clusters. Cluster 1, Cluster 2, and Cluster 3 is convergence. Anomaly data in Membership Cluster $1\left(\mathrm{x}_{22}, \mathrm{x}_{23}, \mathrm{x}_{33}\right)$. Anomaly data in Membership Cluster $2\left(\mathrm{x}_{22}, \mathrm{x}_{23}, \mathrm{x}_{29}, \mathrm{x}_{33}\right)$. Anomaly data in Membership Cluster $3\left(\mathrm{x}_{29}\right)$. Consistency data on K-Means $\left(\mathrm{C}_{1}=\right.$ $\left.72.73 \%, C_{2}=93.75 \%, C_{3}=100 \%\right)$. Consistency data on Fuzzy C-Means $\left(C_{1}=100 \%, C_{2}\right.$ $=88.33 \%, \mathrm{C}_{3}=87.50 \%$ ). Rice Productivity in Cluster 1 (decrease), Cluster 2 (decrease, except 3 provinces), and Cluster 3 (increase, except 1 province). It is meaning that Rice Productivity on Cluster 1 and Cluster 2 is low productivity. From 34 provinces, there is 10 provinces $=$ high productivity $(10 / 34 * 100 \%=29.41 \%)$ and 24 provinces $=$ low productivity $(24 / 34 * 100 \%=70.59 \%)$. Majority membership cluster in K-Means is $\mathrm{C}_{2}$ (47.06\%). Majority membership cluster in Fuzzy C-Means is $\mathrm{C}_{2}(52.94 \%)$. Concluded that is majority of rice productivity per province in Indonesia is low. Future work, clustering by using Heuristic Fuzzy Clustering and Hierarchical Clustering.

\section{References}

[1] T. Scaria, G. Stephen and J. Mathew, "Gene expression data analysis using fuzzy c-means clustering technique”, International Journal of Computer Applications, vol. 135 , no. 8, (2016), pp. 33-36.

[2] E. Mehdizadeh and A. Golabzaei, "Electrical fuzzy c-means: a new heuristic fuzzy clustering algorithm", Cogent Engineering, (2016), pp. 1-15.

[3] Z. Cebeci and F. Yildiz, "Comparison of k-means and fuzzy c-means algorithms on different cluster structures", Journal of Agricultural Informatics, (2015), pp. 13-23.

[4] A. Gong, Y. Gao, X. Ma, W. Gong, H. Li and Z. Gao, "An optimized artificial bee colony algorithm for clustering”, International Journal of Control and Automation, vol. 9, no. 4, (2016), pp. 107-116.

[5] A. X. Ye and Y. X. Jin, "A fuzzy c-means clustering algorithm based on improved quantum genetic algorithm", International Journal of Database Theory and Application, vol. 9, no.1, (2016), pp. 227-236.

[6] E. A. S. Brar and K. Singh, "Potato defect detection using fuzzy c-mean clustering based segmentation", Indian Journal of Science and Technology, vol. 9, no. 32, (2016), pp. 1-6.

[7] S. Ghosh and S. K. Dubey, "Comparative analysis of k-means and fuzzy c-means algorithms", International Journal of Advanced Computer Science and Applications, vol. 4, no. 4, (2013), pp. 35-39.

[8] S. Singla and B. S. Khehra, "A comparative study of k-means, fuzzy c-means and possibilistic fuzzy cmeans algorithm on noisy grayscale images", International Journal of Computer Applications, (2016), pp. 1-5.

[9] S. Majumder, S. A. Anand, K. A. Javid and V. Katarwar, "Brain tumor segmentation mechanism by using k mean and fuzzy c means", International Journal of Innovative Research in Computer and Communication Engineering, vol. 4, iss. 4, (2016), pp. 7445-7451.

[10] A.Sheshasayee and P. Sharmila, "Comparative study of fuzzy c means and k means algorithm for requirements clustering", Indian Journal of Science and Technology, vol. 7, no. 6, (2014), pp. 853-857.

[11] D. Bora and A. K. Gupta, "A comparative study between fuzzy clustering algorithm and hard clustering algorithm", International Journal of Computer Trends and Technology, vol.10, no. 2,(2014), pp.08-113.

[12] Widiarina, "Data clustering using k-means dynamic algorithm", Journal of Computer Engineering AMIK BSI, vol. 1, no. 2, (2015), pp. 260-265.

[13] B. N. Prasad, M. Rathore, G. Gupta and T. Singh, "Performance measure of hard c-means, fuzzy cmeans and alternative c-means algorithms", International Journal of Computer Science and Information Technologies, vol. 7 no. 2, (2016), pp. 878-883.

[14] A. F. Nurjanah, and F. Indriani, "Implementation fuzzy c-means on clustering system of paddy variety", Journal Collection of Computer Science, vol. 1, no. 1, (2014), pp. 23-32. 